\title{
The ultraluminous X-ray source bubble in NGC 5585
}

\author{
R. Soria ${ }^{1,2}$, M.W. Pakull ${ }^{3}$, C. Motch $^{3}$, J.C.A. Miller-Jones ${ }^{4}$, A.D. Schwope ${ }^{5}$, \\ R.T. Urquhart ${ }^{6}$, and M.S. Ryan ${ }^{4}$ \\ ${ }^{1}$ College of Astronomy and Space Sciences, University of the Chinese Academy of Sciences, Beijing 100049, China \\ ${ }^{2}$ Sydney Institute for Astronomy, School of Physics A28, The University of Sydney, Sydney, NSW 2006, Australia \\ ${ }^{3}$ Université de Strasbourg, CNRS, Observatoire astronomique, CNRS, UMR 7550,F-67000, Strasbourg, France \\ ${ }^{4}$ International Centre for Radio Astronomy Research, Curtin University, GPO Box U1987, Perth, WA 6845, Australia \\ ${ }^{5}$ Leibniz-Institut für Astrophysik Potsdam (AIP), An der Sternwarte 16, 14482, Potsdam, Germany \\ ${ }^{6}$ Center for Data Intensive and Time Domain Astronomy, Department of Physics and Astronomy, Michigan State University, East Lansing, MI, USA
}

Accepted 2020 December 3. Received 2020 November 11; in original form 2020 August 17

\begin{abstract}
Some ultraluminous X-ray sources (ULXs) are surrounded by collisionally ionized bubbles, larger and more energetic than supernova remnants: they are evidence of the powerful outflows associated with super-Eddington X-ray sources. We illustrate the most recent addition to this class: a huge $(350 \mathrm{pc} \times 220 \mathrm{pc}$ in diameter) bubble around a ULX in NGC 5585. We modelled the X-ray properties of the ULX (a broadened-disc source with $L_{X} \approx 2-4 \times 10^{39} \mathrm{erg}$ $\mathrm{s}^{-1}$ ) from Chandra and XMM-Newton, and identified its likely optical counterpart in Hubble Space Telescope images. We used the Large Binocular Telescope to study the optical emission from the ionized bubble. We show that the line emission spectrum is indicative of collisional ionization. We refine the method for inferring the shock velocity from the width of the optical lines. We derive an average shock velocity $\approx 125 \mathrm{~km} \mathrm{~s}^{-1}$, which corresponds to a dynamical age of $\sim 600,000$ years for the bubble, and an average mechanical power $P_{\mathrm{w}} \sim 10^{40} \mathrm{erg} \mathrm{s}^{-1}$; thus, the mechanical power is a few times higher than the current photon luminosity. With Very Large Array observations, we discovered and resolved a powerful radio bubble with the same size as the optical bubble, and a 1.4-GHz luminosity $\sim 10^{35} \mathrm{erg} \mathrm{s}^{-1}$, at the upper end of the luminosity range for this type of source. We explain why ULX bubbles tend to become more radio luminous as they expand while radio supernova remnants tend to fade.
\end{abstract}

Key words: accretion, accretion disks - stars: black holes - X-rays: binaries - shock waves

\section{INTRODUCTION}

In the process of accretion onto a stellar-mass compact object, the relative output of radiative and mechanical power is a function of accretion rate and of other parameters such as the geometry of the inflow and the nature of the compact object. Typical regimes well studied in Galactic X-ray binaries (Fender et al. 2004; Remillard \& McClintock 2006; Fender et al. 2009) are: (i) the low/hard state, where the kinetic power of a collimated jet increasingly dominates over photon emission, for low accretion rates (Fender et al. 2003); and (ii) the disk-dominated thermal state (high/soft state), where the jet is quenched (Meier 2001; Russell et al. 2011) and the accretion flow forms a radiatively efficient standard disk. Over the last decade, a lot of observational and theoretical effort has focused on the properties of accretion at even higher accretion rates, above the classical Eddington limit. X-ray binaries in this super-critical regime

^ Email: rsoria@nao.cas.cn (RS) are generally known as ultraluminous X-ray sources (ULXs: Kaaret et al. 2017; Feng \& Soria 2011). One of the hallmark predictions for the super-critical accretion regime is the presence of strong radiatively-driven outflows, launched from the disk surface (King \& Pounds 2003; Poutanen et al. 2007; Dotan \& Shaviv 2011). Magneto-hydrodynamical simulations (Ohsuga \& Mineshige 2011; Kawashima et al. 2012; Jiang et al. 2014; Ogawa et al. 2017; Narayan et al. 2017) suggest that the massive disk wind creates a lowerdensity funnel along the polar directions; a collimated jet may be launched inside that funnel. The presence of strong outflows has been directly confirmed by X-ray spectroscopic studies of a few nearby ULXs (Pinto et al. 2016; Walton et al. 2016; Pinto et al. 2017; Kosec et al. 2018)

The accretion models and observations cited above suggest that the kinetic power of outflows from super-critical accretors is of the same order of magnitude as the radiative power. One of the most effective ways to identify the presence of strong outflows and measure or constrain their power is to search for large $(\sim 100 \mathrm{pc})$ bubbles of shock-ionized gas around the compact object ("ULX 
bubbles": Pakull \& Mirioni 2002; Wang 2002; Pakull et al. 2006; Ramsey et al. 2006; Soria et al. 2010). The size and expansion velocity of a shock-ionized bubble constrain its kinetic energy (Weaver et al. 1977) and its age, which is a proxy for the duration of the super-critical accretion phase. The flux in diagnostic optical lines (particularly, $\mathrm{H} \beta$ ) is another, independent proxy for the input power that inflates the bubble (Dopita \& Sutherland 1995; Pakull et al. 2010). We have been conducting a long-term program of search, identification and modelling of ULX bubbles in nearby galaxies. In addition to a better understanding of accretion processes in ULXs, modelling the properties of such bubbles provides a template to understand other astrophysical phenomena, such as phases of enhanced nuclear activity in normal galaxies (e.g., Guo \& Mathews 2012; Rampadarath et al. 2018), feedback processes in quasars (e.g., King \& Pounds 2015; Tombesi et al. 2015; Parker et al. 2017), or the role of X-ray binaries and microquasars in cosmic re-ionization (e.g., Mirabel et al. 2011; Fragos et al. 2013; Madau \& Fragos 2017; Douna et al. 2018).

In this paper, we present the first detailed study of a huge $(\approx 350$ by $220 \mathrm{pc}$ ) shock-ionized bubble in the outskirts of the Sd galaxy NGC 5585 (Figure 1), a member of the M 101 group (Tikhonov et al. 2015; Karachentsev \& Makarova 2019), with a star formation rate of $\approx 0.4 M_{\odot} \mathrm{yr}^{-1}$ (James et al. 2004). An accurate distance to NGC 5585 remains elusive, in the absence of Cepheid or tip-of-thered-giant-branch measurements. A series of measurements based on the near-infrared Tully-Fisher relation, listed in the NASA/IPAC Extragalactic Database ${ }^{1}$, suggests an average distance of $\approx 8 \mathrm{Mpc}$ for a Hubble constant of $\approx 74 \mathrm{~km} \mathrm{~s}^{-1} \mathrm{Mpc}^{-1}$ (Riess et al. 2019). We adopt $d=8.0 \mathrm{Mpc}$ in this paper.

The optical bubble was first noted and studied by Matonick \& Fesen (1997); they described it as an "enormous" and "particularly interesting" supernova remnant (SNR). Their optical spectrum (taken with the 2.4-m Hiltner telescope at the Michigan-DartmouthMIT Observatory in 1994 May) shows that the gas is shock-ionized and suggests velocity broadening for the strongest emission lines. This peculiar "SNR" is similar in size and luminosity to another exceptional "SNR" also shown in Matonick \& Fesen (1997), namely NGC 7793-S26. It was proposed by Pakull \& Grisé (2008) that both those ionized bubbles are not SNRs but are instead powered by jets or outflows from a compact object in a super-critical accretion regime. For the NGC 5585 source, this physical interpretation was supported by the discovery of a ULX (henceforth NGC 5585 X-1) in the centre of the bubble (Pakull \& Grisé 2008) ${ }^{2}$. Several other shock-ionized bubbles with similar size $(\sim 100-300 \mathrm{pc})$ have been identified in nearby galaxies as accretion-powered bubbles. In addition to the already mentioned NGC 7793-S26 (Pakull et al. 2010; Soria et al. 2010), the best known are the bubbles around the ULXs NGC 1313 X-2 (Pakull \& Mirioni 2002; Weng et al. 2014), Holmberg IX X-1 (Pakull \& Mirioni 2002; Pakull et al. 2006; Moon et al. 2011), IC 342 X-1 (Cseh et al. 2012).

Here, we investigate the large bubble around NGC 5585 X-1 in more detail. We collected and studied new and archival data in several bands: in the X-ray band with Chandra and XMM-Newton; in the optical band with the Hubble Space Telescope (HST) for imaging and the Large Binocular Telescope $\left(\mathrm{LBT}^{3}\right)$ for spectroscopy; in the

\footnotetext{
1 https://ned.ipac.caltech.edu

2 The name "NGC 5885" instead of NGC 5585 in Pakull \& Grisé (2008) is a typo.

3 The LBT is an international collaboration among institutions in the United States, Italy and Germany. LBT Corporation partners are: The University of Arizona on behalf of the Arizona university system; Istituto Nazionale
}

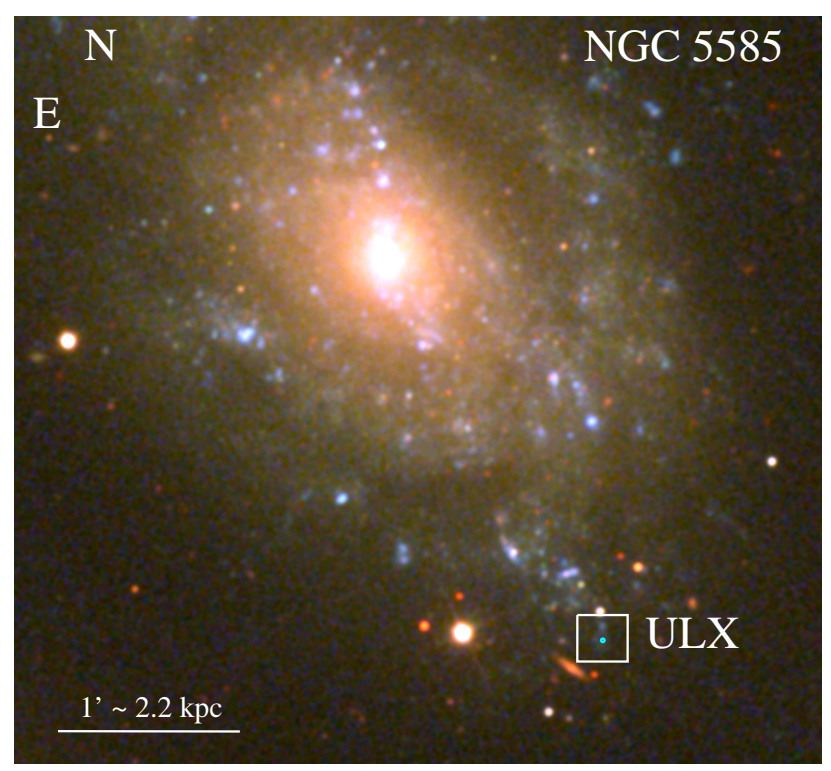

Figure 1. Sloan Digital Sky Survey image of NGC 5585 (red $=i$ band, green $=g$ band, blue $=u$ band), marking the location of the ULX and its bubble. The region inside the white box is displayed in detail in Figure 2.

radio band with the Karl G. Jansky Very Large Array (VLA). We will determine the properties of the central point-like source X-1, the shock velocity of the bubble, its dynamical age and mechanical power. We will show that the ionized nebula is indeed one of the most powerful ULX bubbles in the local universe.

\section{OBSERVATIONS AND DATA ANALYSIS}

\subsection{Chandra}

NGC 5585 was observed by Chandra twice (ObsID $7050=2006$ August 28, and ObsID $19348=2017$ May 31), on both occasions only with a 5-ks exposure time (Table 1). In both observations, the centre of the galaxy was placed at the aimpoint on the S3 chip of the Advanced CCD Imaging Spectrometer array (ACIS). We downloaded the public-archive data, and analyzed them with the Chandra Interactive Analysis of Observations (CIAO) software version 6.12 (Fruscione et al. 2006), with calibration database version 4.9.1. We used the task chandra_repro to re-build level-2 event files, and we filtered it with dmcopy. The point-like source NGC5585 X-1 is detected in both observations (Section 3.1); we used specextract to extract background-subtracted spectra from the two epochs. Finally, we fitted the spectra with XSPEC (Arnaud 1986) version 12.11.0, using the Cash statistics, given the low number of counts.

\subsection{XMM-Newton}

We observed NGC 5585 twice with the European Photon Imaging Camera (EPIC), on 2015 June 19 and 2015 June 21, on both occasions with a duration of $39 \mathrm{ks}$ including overheads (live time: $37 \mathrm{ks}$

di Astrofisica, Italy; LBT Beteiligungsgesellschaft, Germany, representing the Max-Planck Society, the Astrophysical Institute Potsdam, and Heidelberg University; The Ohio State University, and The Research Corporation, on behalf of The University of Notre Dame, University of Minnesota and University of Virginia. 

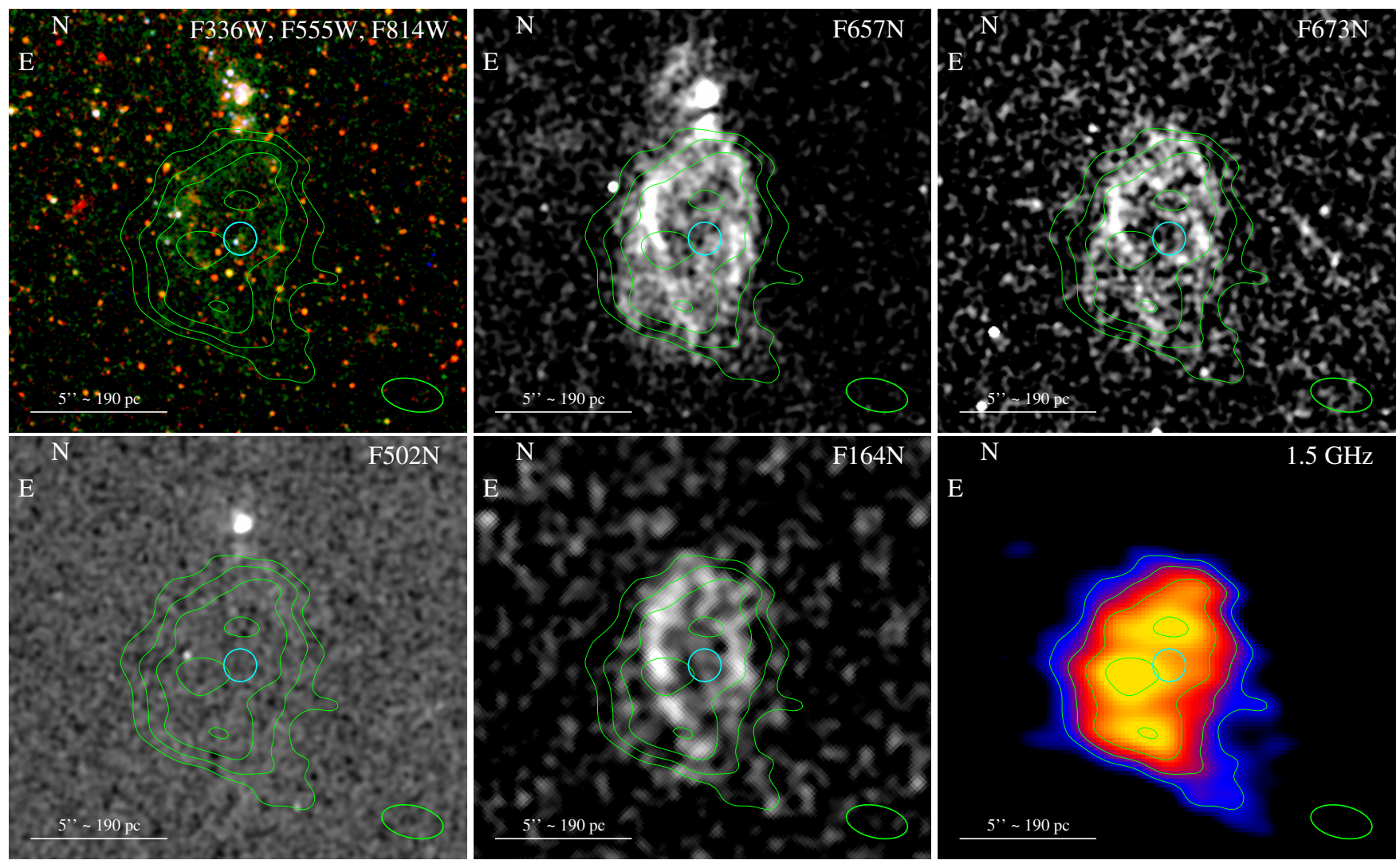

Figure 2. Top row, left panel: HST/WFC3 image of the field around NGC $5585 \mathrm{X}-1$, in the broad-band filters F814W (red), F555W (green) and F336W (blue). The cyan circle marks the location of the ULX (determined from the Chandra detection), and has a radius of $0^{\prime \prime} .6$ (astrometric uncertainty). The green contours show the 1.5-GHz radio emission from the VLA observations; more specifically, they represent flux densities of $2^{n / 2}$ times the local rms noise level, with $n=$ 4, 5, 6 and 7 (i.e., the lowest contour is a 4- $\sigma$ detection). The green ellipse represents the VLA beam, with major axes of $2^{\prime \prime} .28 \times 1^{\prime \prime} .18$, and Position Angle of $78^{\circ} .0$. Top row, middle panel: continuum-subtracted image in the HST/WFC3 F657N filter (the continuum was a linear combination of the F555W and F814W images), which includes $\mathrm{H} \alpha$ and [N II] $\lambda \lambda 6548,6584$. The cyan circle, green ellipse and green contours are as in the previous panel. Top row, right panel: continuum-subtracted image in the HST/WFC3 F673N filter (the continuum was the same linear combination of F555W and F814W), which covers [S II] $\lambda \lambda 6716,6731$. The cyan circle, green ellipse and green contours are as in the previous panels. Bottom row, left panel: continuum-subtracted image in the HST/WFC3 F502N filter (F555W was used for the continuum), which covers [O III] 25007 . The cyan circle, green ellipse and green contours are as in the previous panels. Bottom row, middle panel: continuum-subtracted image in the HST/WFC3 F164N filter (F160W was used for the continuum), which covers [Fe II] $\lambda 16440$. The cyan circle, green ellipse and green contours are as in the previous panels. Bottom row, right panel: false-colour 1.5-GHz VLA image with associated flux-density contours and ULX position marked by the cyan circle.

for MOS1 and MOS2, $32 \mathrm{ks}$ for pn). We reduced the data with the Science Analysis Software (sas) version 17.0.0; we used the sas tasks epproc and emproc to re-build event files for pn and MOS, respectively.

We filtered the event files to remove intervals of high particle background. In the first observation, the background level was very low, and the whole exposure can be used for subsequent analysis. In the second observation, the background was substantially higher, and flaring at the beginning and at the end of the observation. We selected good-time-intervals at PI $>10000$ with a RATE parameter $\leq 0.5$ for MOS 1 and MOS2, and $\leq 1.4$ for the pn, at $10000<\mathrm{PI}<12000$. These thresholds are slightly higher than usually adopted for EPIC data analysis ${ }^{4}$, but they enabled us to make good use of the long non-flaring intervals. After filtering, we obtained a good time interval of $32 \mathrm{ks}$ for MOS1 and MOS2, and $26 \mathrm{ks}$ for pn. We also filtered the event files with the stan-

\footnotetext{
4 https://www.cosmos.esa.int/web/xmm-newton/sas-thread-epicfilterbackground
}

dard conditions "(FLAG==0) \& \& (PATTERN $<=4)$ " for the pn, and "(\#XMMEA_EM \&\& (PATTERN $<=12)$ " for the MOSs (i.e., keeping single and double events).

NGC $5585 \mathrm{X}-1$ was detected in both observations. We defined a circular source region of radius $20^{\prime \prime}$, but with the caveats described in Section 3.1.1, to avoid contamination from a nearby source, and local background regions four times as large as the source region. For each of the two observations, we extracted individual spectra and built associated response and ancillary response files for the pn and MOS cameras with xmmselect; we then combined the pn and MOS spectra and responses of each observation with epicspeccombine, to increase the signal-to-noise ratio of possible line features. We grouped the two spectra to a minimum of 25 counts per bin, for subsequent $\chi^{2}$ spectral fitting with XSPEC (Arnaud 1986) version 12.11.0. 


\section{$2.3 \quad H S T$}

We observed the field of the candidate ULX bubble in several broadband and narrow-band filters (Figures 2, 3), with the Wide Field Camera 3 (WFC3), Ultraviolet and VISible light camera (UVIS, chip 2), and Infrared camera (IR). All observations were taken between 2016 April 30 and May 1. We used about a dozen bright, isolated sources in common with the Gaia Data Release 2 catalog to improve the astrometry of the HST images. Based on the residual scatter after the re-alignment, we estimate that the HST coordinates are accurate within $\approx 0^{\prime \prime} .1$.

We used the broad-band filters to study the point-like optical counterpart to the ULX (Table 3), and the narrow-band filters to study the line emission from the bubble (Table 4). We retrieved calibrated images (.drc files for WFC3-UVIS, .drz files for WFC3IR) from the Mikulski Archive for Space Telescopes. We used Ds9 imaging and photometry tools to measure net count rates from the point-like counterpart and the extended bubble. For the counterpart, we used a source extraction radius of $0^{\prime \prime} .16$ and a local annular background; we converted the count rates to infinite-aperture values using the online tables of encircled energy fractions. We then converted count rates to magnitudes (in the Vega system) and fluxes, using the latest tables of UVIS zeropoints ${ }^{5}$ and IR zeropoints ${ }^{6}$.

For the narrow-band filters, we subtracted the continuum as follows. For F502N, we used the F555W image, properly rescaled to account for the different filter width. Likewise, for F164N we used a rescaled F160W image. For F657N and F673N, we used a linear combination of F555W and F814W images, proportional to their respective filter widths. More specifically: i) we selected a region (radius of $\approx 20^{\prime \prime}$ ) of the UVIS chip rich in stars but without significant diffuse emission; ii) we measured the count rate from that region in each of the narrow-band filters, and in their corresponding broad-band images; iii) we re-normalized the broad-band images so that their count rates in the test region were equal to the count rates in the associated narrow-band images; iv) we subtracted the re-normalized broad-band images from the narrow-band ones; $v$ ) we visually inspected the field around X-1 in the continuum-subtracted narrow-band images to verify that the point-like stars had been properly removed and that any imperfectly-subtracted stellar residuals were negligible (of order of 1 percent) compared with the diffuse nebular emission. We used the fimage subpackage of the FTools software (Blackburn 1995) for these operations, following standard practice.

\subsection{LBT}

We observed the nebula with the LBT at Mt Graham, on 2018 June 12-13. The LBT consists of twin 8.4-m telescopes, but at the time of our observations only one spectrograph, the Multi-Object Double Spectrograph 1 (MODS1), was in operation. In this detector, a dichroic splits the light beam towards the red and blue spectrograph channels; see Pogge, et al. (2010) for a technical description of the instrument. We took blue and red spectra with a $0^{\prime \prime} .6$ slit (Figure 4), oriented at two different Position Angles (PAs). The first orientation was along the major axis of the bubble $\left(\mathrm{PA}=355^{\circ}\right)$, including also the young star clusters at the northern tip of the bubble or just outside it. The second orientation was across the bubble $\left(\mathrm{PA}=65^{\circ}\right)$, in such a way to include the brightest edges of the nebular emission region,

5 http://www.stsci.edu/hst/wfc3/analysis/uvis_zpts/uvis1_infinite

6 http://www.stsci.edu/hst/wfc3/ir_phot_zpt

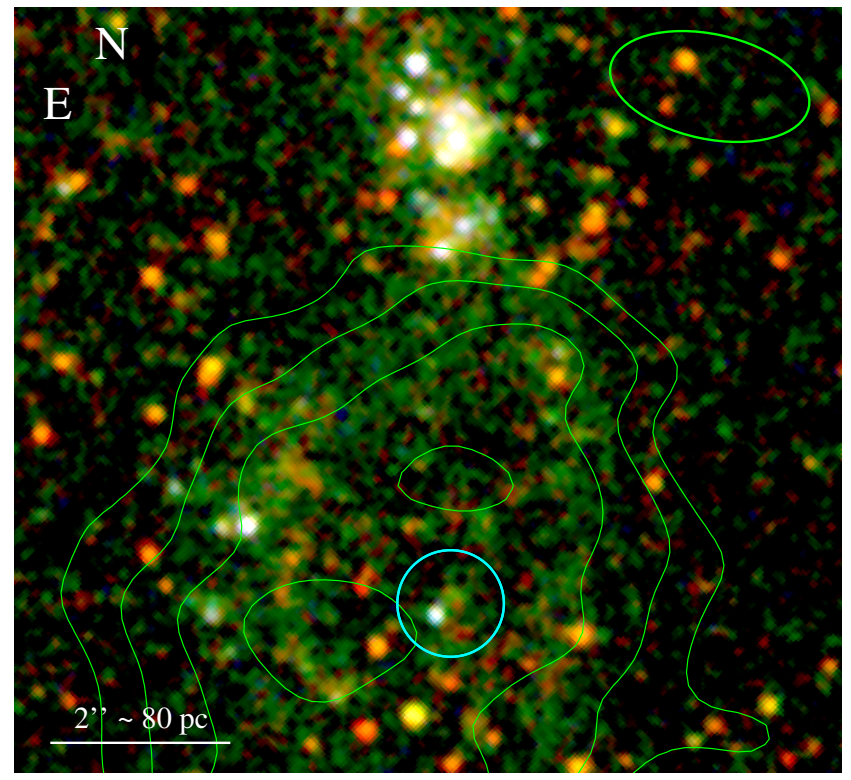

Figure 3. Zoomed-in image of the field around NGC $5585 \mathrm{X}-1$, in the WFC3 broad-band filters F814W, F555W and F336W, with radio contours; notice the single blue star-like object inside the $0^{\prime \prime} .6$ ULX error radius (cyan circle), which we assume to be the optical counterpart of the X-ray source. Notice also the cluster of young stars immediately above the radio bubble. The green contours and green ellipse were defined in Figure 2.

roughly $2^{\prime \prime}$ to the north-east and the south-west of the ULX. For both orientations, the point-like stellar counterpart of NGC 5585 $\mathrm{X}-1$ was presumably on the slit, although no trace can be seen in the two-dimensional spectra because of its faintness.

For the red spectra at both orientations, we used the G670L grating (250 lines/mm), which gives us a resolution of 2300 at 7600 $\AA$ (nominal dispersion of $0.845 \AA$ per pixel). For the blue spectra, we used the G400L grating (400 lines $/ \mathrm{mm}$ ), with a resolution of 1850 at $4000 \AA$ (nominal dispersion of $0.51 \AA$ per pixel). At both slit positions, six exposures of $800 \mathrm{~s}$ each were obtained.

We bias-subtracted and flat-fielded the raw data with the reduction software modsCCDRed Version 2.0.1, provided by Ohio State University ${ }^{7}$. We used the Munich Image Data Analysis System (MIDAs: Warmels 1992) for spectral trimming and to determine the wavelength solution. Lines from an Argon lamp yielded the blue channel calibration while the red channel was wavelength calibrated using a mix of $\mathrm{Ne}, \mathrm{Hg}, \mathrm{Kr}, \mathrm{Xe}$ and $\mathrm{Ar}$ lines. The spectrophotometric standard HZ44 provided an absolute flux calibration common to the blue and red spectra. For further data analysis, such as measurements of line fluxes, line widths and central positions, we used software from both MIDAs (in particular, the integrate/line task) and from the Image Reduction and Analysis Facility (IRAF) Version 2.16 (in particular, the splot sub-package).

\subsection{VLA}

We observed the field around NGC 5585 X-1 four times with the VLA between 2015 September 5 and 18 (project ID 15A142). The phase centre was placed at RA $=14^{h} 19^{m} 39^{s} .400$, Dec $=+56^{\circ} 41^{\prime} 52^{\prime \prime} .70$, slightly offset $\left(\approx 15^{\prime \prime}\right)$ from the target. A total

7 http://www.astronomy.ohio-state.edu/MODS/Software/modsCCDRed/ 


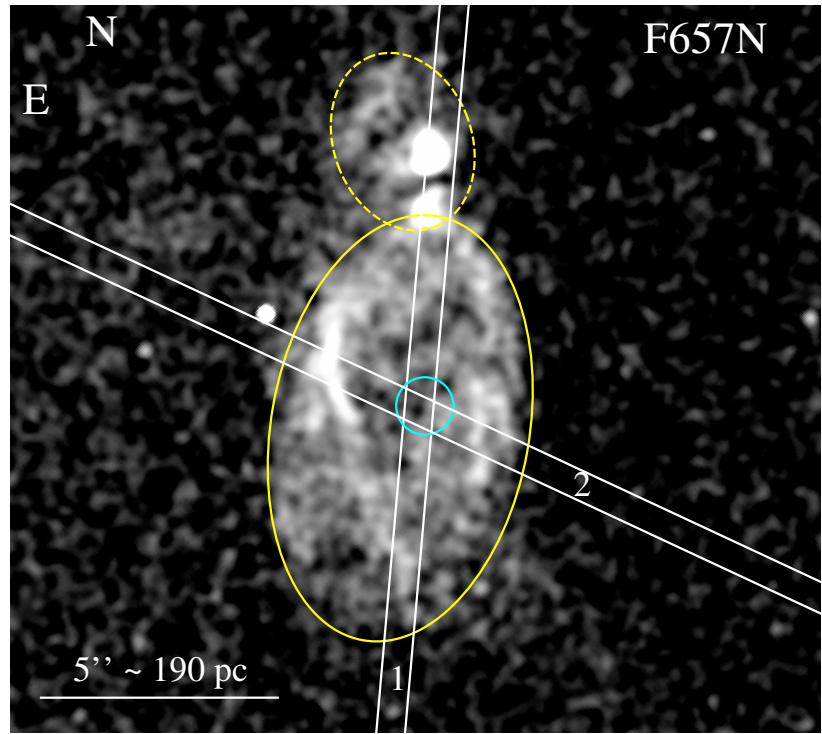

Figure 4. Schematic interpretation of the ionized optical nebula, based on the observed line ratios: the region inside the solid yellow ellipse is the true ULX bubble, dominated by collisional ionization, and with associated synchrotron radio emission; the upper region inside the dashed ellipse is a photo-ionized H II region (ionized by the young stars immediately above the ULX bubble). The positions of the slit in our two LBT observations are overplotted (Obs1 running roughly North-South and Obs2 running roughly East-West). The cyan circle is the location of X-1 (its error radius is $0^{\prime \prime} .6$ ).

integrated time on source of $\approx 3 \mathrm{hr}$ was achieved. For each of the observations, the telescope was in its extended A configuration. Data were taken in the $\mathrm{L}$ band, with two contiguous $512-\mathrm{MHz}$ bands observed simultaneously, spanning the $1-2 \mathrm{GHz}$ frequency range. We used 3C 286 as the bandpass/flux calibrator, while J1400+6210 was used as the phase calibrator.

We used the Common Astronomy Software Application (CASA; McMullin et al. 2007) to perform gain and phase calibration. All four observations were stacked and imaged using the CLEAN algorithm, with Briggs weighting set at a robust value of 1 . The final cleaned image (Figure 2, bottom right panel) has a Gaussian restoring beam of $2^{\prime \prime} .28 \times 1^{\prime \prime} .18$ with a position angle of $77^{\circ} .98$ East of North, and a local rms noise level of $17 \mu \mathrm{Jy}_{\text {beam }}{ }^{-1}$.

\section{MAIN RESULTS}

\subsection{X-ray properties of NGC 5585 X-1}

\subsubsection{Identification of $X-1$ and of a nearby sources}

Our Chandra and XMM-Newton study confirmed the presence of a point-like source with an X-ray luminosity of a few times $10^{39}$ $\mathrm{erg} \mathrm{s}^{-1}$, located near the centre of the optical bubble. We can easily discount the possibility of a chance coincidence with a background $\mathrm{X}$-ray source. At the flux level observed from X-1 ( $f_{\mathrm{X}} \approx 3 \times 10^{-13}$ erg cm $\mathrm{cm}^{-2} \mathrm{~s}^{-1}$, as derived later in this Section), we expect $\sim 2-4$ background X-ray sources (Cappelluti et al. 2009; Luo et al. 2017) per square degree. This corresponds to a probability of $\sim 1 \%$ to detect one such X-ray source projected behind the whole D25 of NGC 5585. Then, the probability that such source happened to be randomly projected in the centre of this exceptional optical/radio bubble would be another three orders of magnitude smaller than that.

We then analyzed the possibility of confusion with other sources. From the XMM-Newton/EPIC-MOS1 and Chandra/ACIS $\mathrm{X}$-ray contours (Figure 5), there is clearly another (much fainter) source $\approx 18^{\prime \prime}$ north-east of X-1. It is listed as CXOU J141940.7+564150 in the Chandra Source Catalog (Evans et al. 2010, 2019), and may be an ordinary high mass X-ray binary in the young stellar population of NGC 5585. In Chandra, that source is clearly not a problem for the analysis of X-1. Instead, in XMM-Newton, it contaminates the emission from X-1 slightly, particularly in EPIC-pn, which has lower spatial resolution than the MOS. We carefully assessed and tried to mitigate the contamination. From our modelling of the Chandra data, we estimate an average $0.3-10 \mathrm{keV}$ absorbed flux $\approx 1.1 \times 10^{-14} \mathrm{erg} \mathrm{cm}^{-2} \mathrm{~s}^{-1}$ for CXOU J141940.7+564150 (a factor of 30 fainter than X-1, as we shall see later). In the XMM-Newton data, the flux was a factor of two higher (although more difficult to estimate, given the stronger source X-1 nearby); both the 3XMM Data Release 7 Serendipitous Source Catalogue from Stacks (Traulsen et al. 2019) and the 3XMM Data Release 8 Serendipitous Source Catalogue (Rosen et al. 2016) list a $0.2-12 \mathrm{keV}$ flux $\approx 2.3-2.4 \times 10^{-14} \mathrm{erg} \mathrm{cm}^{-2} \mathrm{~s}^{-1}$. [Curiously, the same source is no longer listed in the 4XMM Data Release 9 Serendipitous Source Catalogue (Webb et al. 2020) nor in the 4XMM Data Release 9 Serendipitous Source Catalogue from Stacks (Traulsen et al. 2020).] For a large X-1 source extraction circle $\left(\approx 30^{\prime \prime}-40^{\prime \prime}\right.$ radius), CXOU J141940.7+564150 would add $\approx 4-7 \%$ of the observed EPIC flux in the two XMM-Newton observations, which is a significant error and may even affect the observed spectral shape. Thus, we restricted the X-1 extraction radius to $20^{\prime \prime}$, and we placed a small exclusion circle $\left(10^{\prime \prime}\right.$ radius $)$ around the position of CXOU J141940.7+564150. With this careful choice of X-1 source region, the estimated flux contamination is reduced to $\approx 1-$ $1.5 \%$, well below the level of other observational and systematic uncertainties.

\subsubsection{Spectral properties of X-1 from XMM-Newton}

We start our analysis from the XMM-Newton/EPIC spectra, which have a higher signal to noise ratio than the Chandra spectra, and allow a more complex modelling. For every spectral model, we included a photo-electric absorption component (tbabs in XSPEC, with the abundances of Wilms et al. 2000) fixed at the Galactic line-ofsight value of $2.7 \times 10^{20} \mathrm{~cm}^{-2}$, and an additional intrinsic tbabs component left as a free parameter. A simple power-law model is not a good fit (Table 2), because the spectra have significant intrinsic curvature in the EPIC band. A standard disk-blackbody model (Shakura \& Sunyaev 1973; Makishima et al. 1986) provides a better fit (Figure 6: $\chi_{v}^{2}=168.5 / 155$ for June 19 and $\chi_{v}^{2}=126.7 / 117$ for June $21)$, with a characteristic inner disk radius $R_{\text {in }}(\cos \theta)^{1 / 2} \approx(60 \pm 5)$ $\mathrm{km}$ (June 19) or $R_{\text {in }}(\cos \theta)^{1 / 2} \approx(57 \pm 7) \mathrm{km}$ (June 21). This is consistent with the innermost stable circular orbit around a stellar-mass black hole (60 km in case of a non-spinning $7-M_{\odot}$ black hole).

However, the standard disk solution has a rather high peak temperature $\left(k T_{\text {in }} \approx 1.4 \mathrm{keV}\right.$ in both observations) and a de-absorbed $0.3-10 \mathrm{keV}$ isotropic luminosity $\approx 2-3 \times 10^{39} \mathrm{erg} \mathrm{s}^{-1}$. Taken together, high disk temperature and luminosity formally correspond to super-Eddington mass accretion rates, which are not self-consistent with the standard (sub-Eddington) disk-blackbody model. At such high accretion rates, we expect the disk spectrum to be modified by Comptonization and/or energy advection. Therefore, we generalize 
the disk model in our spectral fits to take into account those two possibilities.

First, we approximate a Comptonized disk spectrum with the model simpl $\times$ diskbb. The convolution model simpl (Steiner et al. 2009) includes a fitting parameter for the fraction of seed photons upscattered into a power-law component. The standard disk spectrum corresponds to the case in which the fraction of scattered photons is zero. In our case, the spectrum from the first $X M M$ Newton observation has a best-fitting scattering fraction of 0.26 , but is barely higher than zero at the $90 \%$ confidence limit (scattering fraction of 0.02). In the (more noisy) second observation, the scattering fraction is consistent with zero at the $90 \%$ confidence level. In both observations, the inner-disk colour temperature $k T_{\text {in }} \approx 1.3 \mathrm{keV}$ and the inner radius $R_{\text {in }}(\cos \theta)^{1 / 2} \approx 70-80 \mathrm{~km}$. We conclude that although the simpl $\times$ diskbb model is more physical than the pure diskbb model, in statistical terms the improvement is marginal at best.

Another model successfully applied to many ULXs (Gladstone et al. 2009) consists of a low-temperature disk component ( $k T_{\text {in }} \approx$ $0.1-0.3 \mathrm{keV}$ ) and a warm corona (electron temperature $k T_{e} \approx 1.5-3$ $\mathrm{keV})$. Generally speaking, this model is most suitable when there are two curvature features in the X-ray spectrum: one below $1 \mathrm{keV}$ (associated with a "soft excess") and one around 4-6 keV (highenergy roll-over). We tried to apply this type of model to X-1, using diskir (Gierliński et al. 2008, 2009) in XSPEC. In both XMMNewton spectra, we find no local $\chi^{2}$ minimum corresponding to this class of cool-disk solutions. The statistically favoured diskir solution consists again of a dominant disk component with $k T_{\text {in }} \approx 1.3 \mathrm{keV}$ and $R_{\text {in }}(\cos \theta)^{1 / 2} \approx 70 \mathrm{~km}$, and only a marginal Comptonized component, with a few percent of flux in the high-energy tail. In fact, the Comptonized component is consistent with zero at the $90 \%$ confidence level in the June 21 spectrum. The Comptonized tail is too faint to constrain $k T_{e}$ in either spectrum. Thus, the results of the diskir model are in perfect agreement with what we found with the simpl Comptonization model. We will use the diskir model again later (Section 3.2) to match the X-ray and optical luminosities.

Next, we tried fitting the two spectra with another modified disk model, suitable to super-Eddington accretion: the "slim disk" solution (Abramowicz et al. 1988; Kato et al. 1998; Watarai et al. 2000, 2001; Vierdayanti et al. 2008), which includes the effects of advection and radiation trapping. For practical fitting purposes, the slim disk solution is well approximated by the $p$-free disk model (diskpbb in XsPEC: Mineshige et al. 1994; Kubota et al. 2005), that is a less radiatively efficient disk model in which the temperature scales as $T \propto R^{-p}$, with $p<0.75$ (the value associated with the radiatively efficient sub-Eddington disk). X-ray spectral studies of Galactic black holes in outburst have shown (Kubota \& Makishima 2004; Abe et al. 2005) that a standard disk transitions to a slim disk when the peak temperature $k T_{\text {in }} \gtrsim 1.2 \mathrm{keV}$. Both our $X M M$ Newton spectra are well-fitted (Table 2) by the $p$-free disk model, with $p \approx 0.7$ and peak colour temperature $k T_{\text {in }} \approx 1.5-1.6 \mathrm{keV}$. However, the standard solution $p \approx 0.75$ is acceptable within the $90 \%$ confidence limit for both spectra. We also tried fitting both spectra with the same slim-disk model parameters, locked between the two epochs, except for a normalization constant; the constant is fixed at 1.0 for the June 19 spectrum, and has a best-fitting value of $0.80 \pm 0.03$ for the spectrum from June 21 (Table 2). In this case, the best-fitting parameter $p=0.68_{-0.04}^{+0.06}$, just lower than 0.75 at the $90 \%$ confidence level. We conclude that a slim disk may be preferable to a standard disk for physical reasons, but the statistical difference between the two models is very marginal.

In summary, all three models (standard disk, Comptonized disk
Table 1. Summary of our Chandra and XMM-Newton observations.

\begin{tabular}{|c|c|c|c|}
\hline \multicolumn{4}{|c|}{ Chandra/ACIS } \\
\hline ObsID & Obs. Date & $\begin{array}{l}\text { Exp. Time } \\
\quad(\mathrm{ks})\end{array}$ & $\begin{array}{c}\text { Net Count Rate } \\
\left(\mathrm{ct} \mathrm{s}^{-1}, 0.3-8.0 \mathrm{keV}\right)\end{array}$ \\
\hline 7150 & 2006 Aug 28 & 5.3 & $(4.7 \pm 0.3) \times 10^{-2}$ \\
\hline 19348 & 2017 May 31 & 4.7 & $(2.8 \pm 0.2) \times 10^{-2}$ \\
\hline \multicolumn{4}{|c|}{ XMM-Newton/EPIC } \\
\hline ObsID & Obs. Date & $\begin{array}{l}\text { Exp. Time } \\
\quad(\mathrm{ks})\end{array}$ & $\begin{array}{c}\text { Net Count Rate } \\
\left(\mathrm{ct} \mathrm{s}^{-1}, 0.3-10.0 \mathrm{keV}\right)\end{array}$ \\
\hline \multirow[t]{3}{*}{0762640101} & 2015 Jun 19 & $32.0(\mathrm{pn})$ & $(9.7 \pm 0.2) \times 10^{-2}$ \\
\hline & & $37.1(\operatorname{MOS} 1)$ & $(2.7 \pm 0.1) \times 10^{-2}$ \\
\hline & & 37.1 (MOS2) & $(2.7 \pm 0.1) \times 10^{-2}$ \\
\hline \multirow[t]{3}{*}{0762640201} & 2015 Jun 21 & $25.5(\mathrm{pn})$ & $(7.7 \pm 0.2) \times 10^{-2}$ \\
\hline & & $32.4(\operatorname{MOS} 1)$ & $(2.1 \pm 0.1) \times 10^{-2}$ \\
\hline & & $32.4(\operatorname{MOS} 2)$ & $(2.1 \pm 0.1) \times 10^{-2}$ \\
\hline
\end{tabular}

and slim disk) give similar de-absorbed luminosities: $L_{X} \approx 3 \times 10^{39}$ $\mathrm{erg} \mathrm{s}^{-1}$ on June 19 , and $L_{\mathrm{X}} \approx 2 \times 10^{39} \mathrm{erg} \mathrm{s}^{-1}$ on June 21 , in the $0.3-$ $10 \mathrm{keV}$ band, and peak disk temperatures $\approx 1.3-1.6 \mathrm{keV}$. Luminosity and peak temperature are self-consistent and typical of a mildly super-Eddington regime, sometimes known as the "broadened disc" regime (Sutton et al. 2013).

Estimating the inner radius of a slim disk is less straightforward than for a standard sub-Eddington disk, because it does not coincide with the innermost stable circular orbit (Watarai \& Mineshige 2003). Nonetheless, even for slim disks it is customary to define a "true" inner disk radius $R_{\text {in }} \approx 3.18(\kappa / 3)^{2} r_{\text {in }}$ (Vierdayanti et al. 2008), where $\kappa$ is the spectral hardening factor, $r_{\text {in }}$ is the "apparent" radius from the spectral fit, $r_{\text {in }}(\cos \theta)^{1 / 2}=d_{10}\left(N_{\text {dpbb }}\right)^{1 / 2}, N_{\text {dpbb }}$ is the normalization constant of the diskpbb model in XSPEC, and $d_{10}$ is the distance in units of $10 \mathrm{kpc}$. In standard disks, the hardening factor is $\kappa \approx 1.7$ (Shimura \& Takahara 1995), but for accretion rates at or above the Eddington limit, the hardening factor increases to $\approx 2.5-3$ (Watarai \& Mineshige 2003; Kawaguchi 2003; Shrader \& Titarchuk 2003; Isobe et al. 2012). For a non-rotating black hole, the mass corresponding to a disk radius $R_{\text {in }}$ is then $M \approx$ $1.2 \times R_{\text {in }} c^{2} /(6 G) \approx\left[R_{\text {in }} /(7.4 \mathrm{~km})\right] M_{\odot}$, where the correction factor 1.2 is also a consequence of the slim disk geometry, more specifically of the fact that a slim disk extends slightly inside the innermost stable orbit (Vierdayanti et al. 2008). Our viewing angle $\theta$ is unknown, but if we assume that it is not too close to edgeon, the best-fitting value of $R_{\text {in }} \approx 120 \mathrm{~km}$ (Table 2 ) corresponds to a characteristic mass $\approx 15 M_{\odot}$. Such a system would reach its Eddington limit (and thus show features of the slim disk and/or Comptonized disk regime) when its luminosity reaches $\sim 2 \times 10^{39}$ $\mathrm{erg} \mathrm{s}^{-2}$, consistent with what we find in NGC $5585 \mathrm{X}-1$.

$X M M$-Newton spectra of several other ULXs exhibit features at energies $\sim 0.5-1 \mathrm{keV}$, consistent with emission and absorption lines from warm outflows, at flux levels of a few percent of the continuum flux (Middleton et al. 2014, 2015; Pinto et al. 2016; Urquhart \& Soria 2016; Pinto et al. 2017). For NGC 5585 X-1, we do not find evidence of line residuals in the soft X-ray band, but we cannot 


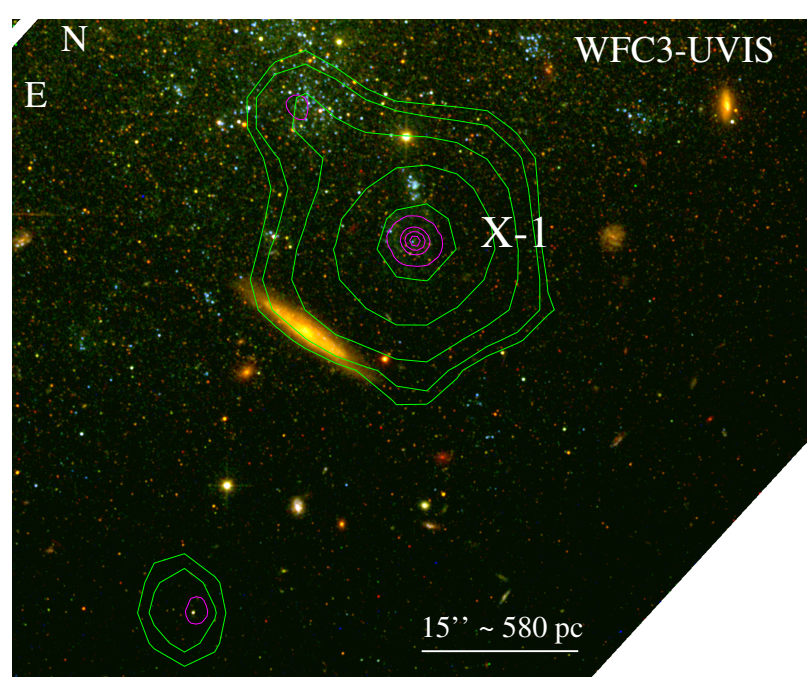

Figure 5. X-ray contours of the XMM-Newton/EPIC-MOS1 (green) and Chandra/ACIS-S (magenta) count rates in the $\mathrm{X}-1$ region, superposed on an HST/WFC3 image $(\mathrm{F} 814 \mathrm{~W}=$ red, F555W = green, F336W = blue). XMMNewton contours are in log scale, from $10^{-5}$ to $10^{-4}$ MOS $1 \mathrm{ct} \mathrm{s}^{-1} \mathrm{arcsec}^{-2}$. Chandra contours are in linear scale, from $1.2 \times 10^{-4}$ to $6.4 \times 10^{-3}$ ACIS-S $\mathrm{ct} \mathrm{s}^{-1} \mathrm{arcsec}^{-2}$. The white corner at the bottom right of the image is simply the edge of the WFC3-UVIS chip. In XMM-Newton (but not in Chandra), $\mathrm{X}-1$ is slightly contaminated (at a few percent level) by another source located $\approx 18^{\prime \prime}$ to the north-east (see Section 3.1.1). Another faint X-ray source is detected both by XMM-Newton and by Chandra, $\approx 40^{\prime \prime}$ south-east of X-1, but it does not create any contamination issue. Its blue optical counterpart suggests that it is a background quasar. The correspondence between the optical and X-ray position of this background source confirms that the Chandra/HST astrometric alignment is better than $\approx 0^{\prime \prime} .5$ (in turn, the HST astrometry is aligned to the Gaia astrometry within $\approx 0^{\prime \prime} .1$ ). This supports our identification of the point-like optical counterpart of X-1 (Section 3.2).

draw strong conclusions because of the relatively low number of counts (Table 1). We tried adding an optically thin thermal plasma component (mekal in XSPEC) to the diskpbb fit, but it does not improve the fit, and the mekal normalization is consistent with 0 within the $90 \%$ confidence limit. If we fix the mekal temperature to $k T=0.7 \mathrm{keV}$, we find a $90 \%$ upper limit of $\approx 6 \times 10^{37} \mathrm{erg} \mathrm{s}^{-1}$ for the 0.3-10 keV luminosity of the thermal plasma emission; for a fixed temperature $k T=1.0 \mathrm{keV}$, the $90 \%$ upper limit is $\approx 10^{38} \mathrm{erg} \mathrm{s}^{-1}$. We also note that the intrinsic column density is low, $N_{\mathrm{H} \text {,int }} \approx 10^{21}$ $\mathrm{cm}^{-2}$. Taken together, the lack of residual line features and the low photo-electric absorption suggest that NGC $5585 \mathrm{X}-1$ is not seen through a dense wind (either because of face-on orientation or because the wind is currently weak).

\subsubsection{Spectral properties of X-1 from Chandra}

The two Chandra observations do not have enough counts $(\approx 250$ net counts for the 2006 data and $\approx 130$ for the 2017 data) for complex, individual spectral modelling. We regrouped each spectrum to 1 count per bin and fitted it with a power-law model, using the Cash statistics (Cash 1979). We find that all the parameters (column density, photon index, normalization) are consistent between the two epochs (Table 3); the lower count rate seen in the 2017 observation (Table 1) is only due to the loss of soft X-ray sensitivity in the ACIS detector. Thus, we refitted the two spectra simultaneously
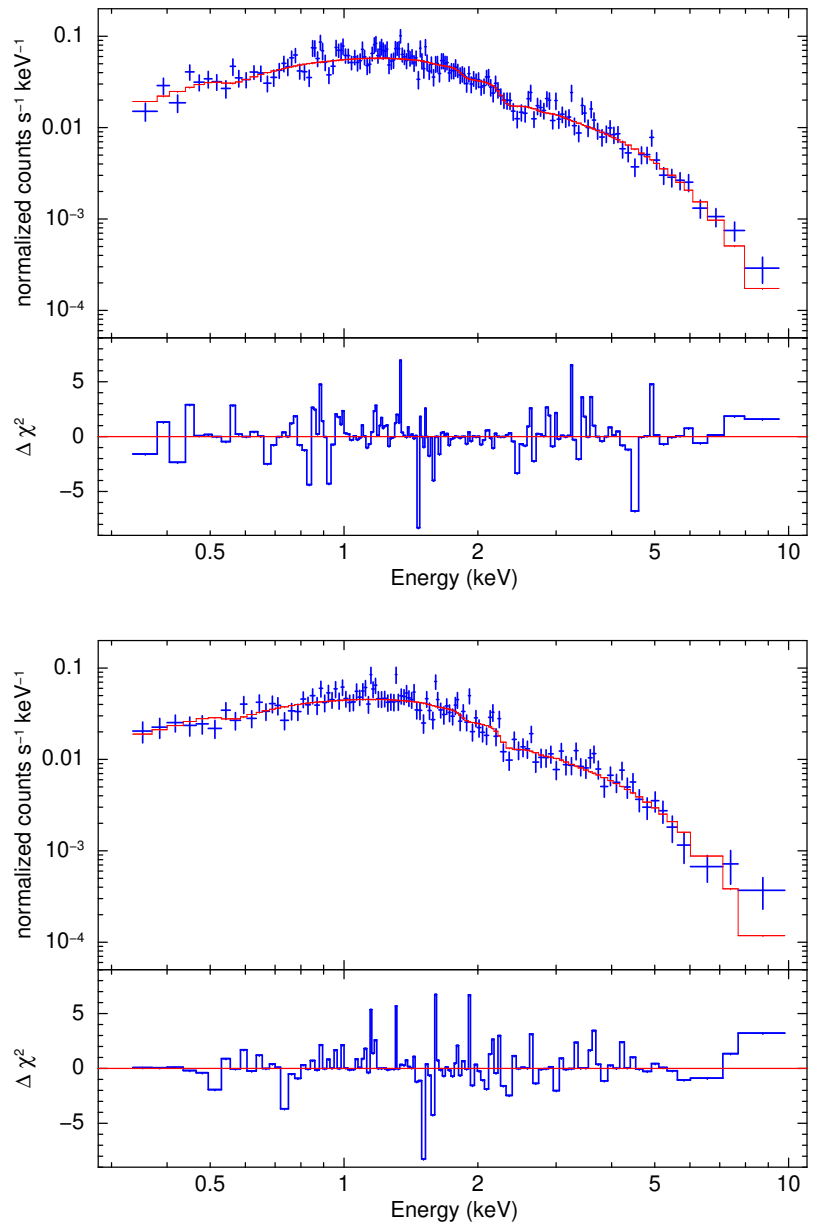

Figure 6. Top panel: $X M M$-Newton/EPIC spectral datapoints and $\chi^{2}$ residuals, from the 2015 June 19 observation of NGC 5585 X-1, fitted with a standard disk-blackbody model (diskbb in XSPEC). The best-fitting inner disk temperature is $T_{\text {in }}=(1.44 \pm 0.07) \mathrm{keV}$, and the inferred inner disk radius is $R_{\text {in }}(\cos \theta)^{1 / 2} \approx(60 \pm 5) \mathrm{km}$. Bottom panel: as in the top panel, for the spectrum obtained in the 2015 June 21 observation; here, $k T_{\text {in }}=(1.39 \pm 0.09)$ $\mathrm{keV}$, and $R_{\text {in }}(\cos \theta)^{1 / 2} \approx(57 \pm 7) \mathrm{km}$. See Table 2 for a more detailed list of spectral parameters, fluxes and luminosities.

with an absorbed diskbb model (Figure 7), and locked all parameters between the two epochs, that is we assumed that the emission was identical. This simultaneous slim disk model has a better $\mathrm{C}$ statistics (227.9 for 281 degrees of freedom) than the independent power-law models (236.8 for 278 degrees of freedom). The bestfitting peak colour temperature is $k T_{\text {in }} \approx(1.2 \pm 0.2) \mathrm{keV}$ (Table 3 ). We used the diskbb model to estimate the observed flux and the de-absorbed luminosity (Table 3 ); the latter is $L_{X} \approx 3.6 \times 10^{39} \mathrm{erg}$ $\mathrm{s}^{-1}$, slightly higher than during the 2015 XMM-Newton observations, but still perfectly consistent with a mildly super-Eddington regime. Irregular flux variability of the amplitude seen between the four XMM-Newton and Chandra observations is very common in persistently active ULXs (Weng \& Feng 2018). The inferred inner disk radius is $R_{\text {in }}(\cos \theta)^{1 / 2} \approx(100 \pm 30) \mathrm{km}$, consistent with the $X M M$-Newton results.

As mentioned earlier (Section 3.1.2), at luminosities $\gtrsim 10^{39}$ $\mathrm{erg} \mathrm{s}^{-1}$ and peak disk temperatures $\gtrsim 1.2 \mathrm{keV}$, theoretical models suggest that the disk emission becomes significantly modified by Comptonization and/or energy advection. As we did with the XMM- 


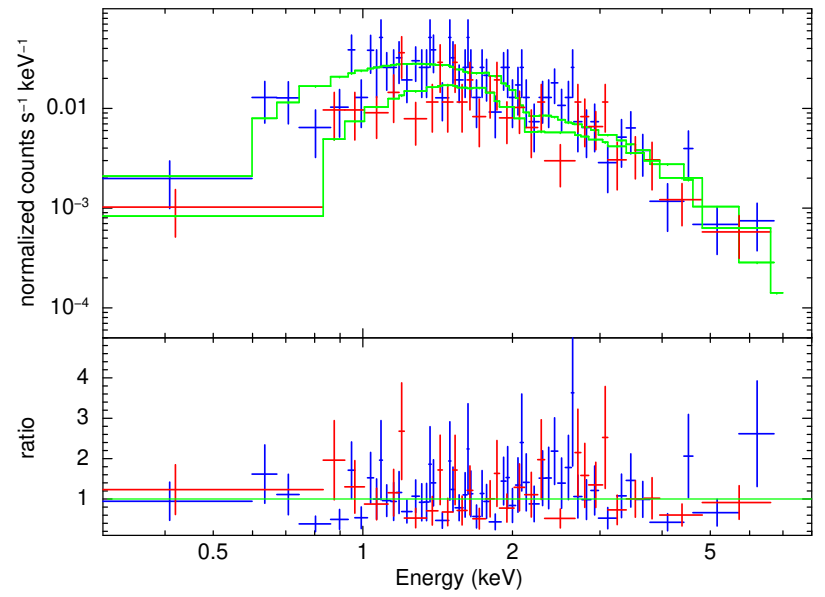

Figure 7. Simultaneous fit to the Chandra/ACIS spectra of NGC 5585 X-1 from 2006 (blue datapoints) and 2017 (red datapoints), with a standard diskblackbody model, and parameters locked between the two epochs. Here, $k T_{\text {in }}=(1.2 \pm 0.2) \mathrm{keV}$, and $R_{\text {in }}(\cos \theta)^{1 / 2} \approx(100 \pm 30) \mathrm{km}$. See Table 3 for the parameter values and luminosity.

Newton spectrum, we tried convolving the diskbb spectrum with the simpl model, but there is no improvement in $\mathrm{C}$ statistics, and the scattering fraction is consistent with zero. We also tried the $p$-free model diskpbb, but again there is no statistical improvement, and the $p$ parameter is consistent with 0.75 .

\subsection{Point-like optical counterpart}

The short exposure times and the moderately off-axis location of NGC 5585 X-1 in both Chandra observations are the main reasons why we cannot substantially improve the astrometric solution of the X-ray images using optical/X-ray associations. Thus, we took the default astrometry of the ACIS event files, and determined the centroid of X-1 in both observations. We obtain a position R.A. $(\mathrm{J} 2000)=14^{h} 19^{m} 39^{s} .38$, Dec. $(\mathrm{J} 2000)=56^{\circ} 41^{\prime} 37^{\prime \prime} .7$ (the same in both observations), with a $90 \%$ error radius of $0^{\prime \prime} .6$. (The $90 \%$ uncertainty radius of an individual Chandra observation is $\approx 0^{\prime \prime} .8^{8}$, but here we are taking the average of two observations.) There is only one bright, blue optical source (Figure 3) that stands out inside the X-ray error circle, in the HST images: it is located at R.A. $(\mathrm{J} 2000)=14^{h} 19^{m} 39^{s} .41$, Dec. $(\mathrm{J} 2000)=56^{\circ} 41^{\prime} 37^{\prime \prime} .6$ (error radius of $\left.0^{\prime \prime} .1\right)$.

We looked for other Chandra sources with an obvious pointlike HST counterpart, which could help us refine the X-1 astrometry, from their relative offsets. There is an X-ray source (listed as CXOGSG J141941.9+564101 in Wang et al. 2016) coincident with an obvious, bright point-like HST counterpart (a background quasar), about $40^{\prime \prime}$ south-east of X-1 (Figure 5); however, it only has 13 ACIS counts scattered within a $2^{\prime \prime}$ radius, and we cannot confidently fit its central position to better than about $0^{\prime \prime} .5$. There is another faint Chandra source about $82^{\prime \prime}$ north-west of X-1, clearly associated with another likely quasar in the HST images, at R.A.(J2000) $=14^{h} 19^{m} 30^{s} .51$, Dec. $(\mathrm{J} 2000)=56^{\circ} 42^{\prime} 13^{\prime \prime} .1$; but it has only 6 ACIS counts, so again we cannot determine the X-ray position with great accuracy. Nonetheless, the coincidence between those two other faint sources and their $H S T$ counterparts within about $0^{\prime \prime} .5$

8 https://cxc.harvard.edu/cal/ASPECT/celmon/
Table 2. Best-fitting parameters of the XMM-Newton/EPIC spectra of X-1. The Galactic absorption is fixed at $N_{\mathrm{H}, \mathrm{Gal}}=2.7 \times 10^{20} \mathrm{~cm}^{-2}$.

\begin{tabular}{|c|c|c|}
\hline \multirow[t]{2}{*}{ Model Parameters } & \multicolumn{2}{|c|}{ Values } \\
\hline & 2015 June 19 & 2015 June 21 \\
\hline \multicolumn{3}{|c|}{$t b a b s \times t b a b s \times p o$} \\
\hline$N_{\mathrm{H}, \text { int }}\left(10^{22} \mathrm{~cm}^{-2}\right)$ & $0.29_{-0.03}^{+0.03}$ & $0.24_{-0.04}^{+0.04}$ \\
\hline$\Gamma$ & $1.88_{-0.06}^{+0.07}$ & $1.85_{-0.09}^{+0.09}$ \\
\hline$N_{\text {po }}^{a}$ & $8.5_{-0.6}^{+0.6}$ & $6.3_{-0.6}^{+0.6}$ \\
\hline$\chi^{2} / \mathrm{dof}$ & $234.7 / 155(1.51)$ & $165.0 / 117(1.41)$ \\
\hline \multicolumn{3}{|c|}{ tbabs $\times$ tbabs $\times$ simpl $\times$ diskbb } \\
\hline$N_{\mathrm{H}, \text { int }}\left(10^{22} \mathrm{~cm}^{-2}\right)$ & $0.07_{-0.02}^{+0.02}$ & $0.04_{-0.02}^{+0.03}$ \\
\hline$\Gamma$ & $1.1_{-0.1}^{+5.0}$ & {$[1.1]$} \\
\hline FracScatt & $0.26_{-0.24}^{+0.08}$ & $<0.41$ \\
\hline$k T_{\text {in }}(\mathrm{keV})$ & $1.29_{-0.31}^{+0.13}$ & $1.30_{-0.18}^{+0.17}$ \\
\hline$N_{\mathrm{dbb}}\left(10^{-3} \mathrm{~km}^{2}\right)^{b}$ & $7.4_{-3.1}^{+3.0}$ & $5.1_{-2.2}^{+5.4}$ \\
\hline$R_{\mathrm{in}} \sqrt{\cos \theta}(\mathrm{km})^{c}$ & $82_{-19}^{+15}$ & $68_{-17}^{+30}$ \\
\hline$\chi^{2} / \mathrm{dof}$ & $165.2 / 153(1.08)$ & $126.2 / 116(1.09)$ \\
\hline \multicolumn{3}{|c|}{$t b a b s \times t b a b s \times$ diskpbb } \\
\hline$N_{\mathrm{H}, \text { int }}\left(10^{22} \mathrm{~cm}^{-2}\right)$ & $0.11_{-0.05}^{+0.02}$ & $0.06_{-0.06}^{+0.06}$ \\
\hline$k T_{\text {in }}(\mathrm{keV})$ & $1.58_{-0.16}^{+0.21}$ & $1.46_{-0.19}^{+0.27}$ \\
\hline$p$ & $0.68_{-0.06}^{+0.07}$ & $0.71_{-0.09}^{+0.15}$ \\
\hline$N_{\mathrm{dpbb}}\left(10^{-3} \mathrm{~km}^{2}\right)^{b}$ & $2.2_{-1.1}^{+2.2}$ & $2.6_{-1.6}^{+4.0}$ \\
\hline$R_{\mathrm{in}} \sqrt{\cos \theta}(\mathrm{km})^{d}$ & $119_{-35}^{+48}$ & $128_{-50}^{+79}$ \\
\hline$\chi^{2} /$ dof & $166.2 / 154(1.08)$ & $126.4 / 116(1.09)$ \\
\hline$f_{0.3-10}\left(10^{-13} \mathrm{erg} \mathrm{cm}^{-2} \mathrm{~s}^{-1}\right)^{e}$ & $3.38_{-0.14}^{+0.13}$ & $2.57_{-0.14}^{+0.14}$ \\
\hline$L_{0.3-10}\left(10^{39} \mathrm{erg} \mathrm{s}^{-1}\right)^{f}$ & $2.91_{-0.18}^{+0.19}$ & $2.13_{-0.18}^{+0.20}$ \\
\hline
\end{tabular}

\begin{tabular}{|c|c|c|}
\hline \multicolumn{3}{|c|}{ tbabs $\times$ tbabs $\times$ const $\times$ diskpbb } \\
\hline$N_{\mathrm{H}, \text { int }}\left(10^{22} \mathrm{~cm}^{-2}\right)$ & \multicolumn{2}{|c|}{$0.09_{-0.04}^{+0.04}$} \\
\hline$k T_{\text {in }}(\mathrm{keV})$ & \multicolumn{2}{|c|}{$1.55_{-0.13}^{+0.15}$} \\
\hline$p$ & \multicolumn{2}{|c|}{$0.68_{-0.04}^{+0.06}$} \\
\hline$C$ & {$[1.00]$} & $0.80_{-0.03}^{+0.03}$ \\
\hline$N_{\mathrm{dpbb}}\left(10^{-3} \mathrm{~km}^{2}\right)^{b}$ & \multicolumn{2}{|c|}{$2.3_{-1.0}^{+1.8}$} \\
\hline$R_{\mathrm{in}} \sqrt{\cos \theta}(\mathrm{km})^{d}$ & \multicolumn{2}{|c|}{$123_{-31}^{+40}$} \\
\hline$\chi^{2} /$ dof & \multicolumn{2}{|c|}{$299.7 / 273(1.10)$} \\
\hline$f_{0.3-10}\left(10^{-13} \mathrm{erg} \mathrm{cm}^{-2} \mathrm{~s}^{-1}\right)^{e}$ & $3.33_{-0.12}^{+0.12}$ & $2.65_{-0.10}^{+0.10}$ \\
\hline$L_{0.3-10}\left(10^{39} \mathrm{erg} \mathrm{s}^{-1}\right)^{f}$ & $2.84_{-0.14}^{+0.16}$ & $2.26_{-0.11}^{+0.13}$ \\
\hline
\end{tabular}

$a$ : units of $10^{-5}$ photons $\mathrm{keV}^{-1} \mathrm{~cm}^{-2} \mathrm{~s}^{-1}$ at $1 \mathrm{keV}$.

b: $N_{\mathrm{dbb}}=\left(r_{\mathrm{in}} / d_{10}\right)^{2} \cos \theta$, where $r_{\mathrm{in}}$ is the apparent inner disk radius in $\mathrm{km}, d_{10}$ the distance to the source in units of $10 \mathrm{kpc}$ (here, $d_{10}=800$ ), and $\theta$ is our viewing angle ( $\theta=0$ is face-on). $N_{\mathrm{dpbb}}$ is defined the same way.

$c: R_{\text {in }} \approx 1.19 r_{\text {in }}$ for a standard disk (Kubota et al. 1998).

$d: R_{\text {in }} \approx 3.18(\kappa / 3)^{2} r_{\text {in }}$ for a slim disk (Vierdayanti et al. 2008; Isobe et al. 2012). Here we assume $\kappa=3$.

$e$ : observed fluxes in the $0.3-10 \mathrm{keV}$ band

$f$ : isotropic de-absorbed luminosities in the $0.3-10 \mathrm{keV}$ band, defined as $4 \pi d^{2}$ times the de-absorbed fluxes. 
Table 3. Best-fitting parameters of the Chandra/ACIS spectra of $\mathrm{X}-1$. As in Table 2, the Galactic absorption is fixed at $N_{\mathrm{H}, \mathrm{Gal}}=2.7 \times 10^{20} \mathrm{~cm}^{-2}$.

\begin{tabular}{lcc}
\hline \hline \multirow{2}{*}{ Model Parameters } & \multicolumn{2}{c}{ Values } \\
& 2006 August 28 & 2017 May 31 \\
\hline & tbabs $\times$ tbabs $\times$ po \\
\hline$N_{\mathrm{H}, \text { int }}\left(10^{22} \mathrm{~cm}^{-2}\right)$ & $0.50_{-0.18}^{+0.21}$ & $0.48_{-0.39}^{+0.47}$ \\
$\Gamma$ & $1.91_{-0.34}^{+0.36}$ & $2.15_{-0.49}^{+0.54}$ \\
$N_{\text {po }}^{a}$ & $1.3_{-0.4}^{+0.6}$ & $1.5_{-0.6}^{+1.2}$ \\
$\mathrm{C}-$ stat/dof & $142.3 / 153(0.93)$ & $94.6 / 125(0.76)$ \\
\hline
\end{tabular}

\begin{tabular}{lc}
\hline \multicolumn{2}{c}{ tbabs $\times$ tbabs $\times$ diskbb } \\
\hline$N_{\mathrm{H}, \text { int }}\left(10^{22} \mathrm{~cm}^{-2}\right)$ & $0.25_{-0.11}^{+0.12}$ \\
$k T_{\text {in }}(\mathrm{keV})$ & $1.18_{-0.15}^{+0.20}$ \\
$N_{\mathrm{dbb}}\left(10^{-3} \mathrm{~km}^{2}\right)^{b}$ & $11.8_{-5.4}^{+8.9}$ \\
$R_{\text {in }} \sqrt{\cos \theta}(\mathrm{km})^{c}$ & $103_{-26}^{+34}$ \\
C-stat $/ \mathrm{dof}$ & $227.9 / 281(0.81)$ \\
$f_{0.3-10}\left(10^{-13} \mathrm{erg} \mathrm{cm}^{-2} \mathrm{~s}^{-1}\right)^{d}$ & $3.9_{-0.5}^{+0.5}$ \\
$L_{0.3-10}\left(10^{39} \mathrm{erg} \mathrm{s}^{-1}\right)^{e}$ & $3.6_{-0.3}^{+0.5}$ \\
\hline
\end{tabular}

a: units of $10^{-4}$ photons $\mathrm{keV}^{-1} \mathrm{~cm}^{-2} \mathrm{~s}^{-1}$ at $1 \mathrm{keV}$.

${ }^{b}: N_{\mathrm{dbb}}=\left(r_{\text {in }} / d_{10}\right)^{2} \cos \theta$, where $r_{\text {in }}$ is the apparent inner disk radius in

$\mathrm{km}, d_{10}$ the distance to the source in units of $10 \mathrm{kpc}$ (here, $d_{10}=800$ ), and $\theta$ is our viewing angle $(\theta=0$ is face-on).

$c: R_{\text {in }} \approx 1.19 r_{\text {in }}$ for a standard disk (Kubota et al. 1998).

$d$ : observed fluxes in the $0.3-10 \mathrm{keV}$ band

$e$ : isotropic de-absorbed luminosities in the $0.3-10 \mathrm{keV}$ band, defined as $4 \pi d^{2}$ times the de-absorbed fluxes.

confirms the good astrometric alignment, supports our identification of the optical counterpart of X-1, and suggests that our error circle of about $0^{\prime \prime} .6$ is a safe, conservative estimate.

The optical brightness and colour of the optical counterpart of X-1 are consistent with those of other "blue" ULX counterparts (Tao et al. 2011; Gladstone et al. 2013). Correcting only for foreground Galactic extinction, we infer absolute brightnesses $M_{F 336 W}=-5.79 \pm 0.04 \mathrm{mag}, M_{F 555 W}=-4.29 \pm 0.03 \mathrm{mag}$, $M_{F 814 W}=-3.65 \pm 0.10 \mathrm{mag}$ (Table 4). The X-ray to optical flux ratio (defined as in Maccacaro et al. 1982) is $\log \left(f_{\mathrm{X}} / f_{V}\right) \approx 2.80$, which is also typical of ULX counterparts (Tao et al. 2011; Gladstone et al. 2013). Of course we are aware that the X-ray to optical flux ratio was not measured simultaneously, and we have no information on how the optical luminosity may vary in response to the observed small changes in X-ray luminosity; but we only need an order-of-magnitude estimate of this ratio to conclude that X-1 is not for example a background AGN (which have typical ratios $\left.-1 \lesssim \log \left(f_{\mathrm{X}} / f_{V}\right) \lesssim 1\right)$.

As is usually the case for blue ULX counterparts, it is hard to tell (especially with only three photometric datapoints) whether the optical source is a young, massive star, or the irradiation-dominated outer accretion disk (or a combination of both). First, we assumed that the emission is entirely from a single donor star. We used the stellar isochrones from the Padova group (Marigo et al. 2017), available online ${ }^{9}$; we assumed a subsolar metal abundance $(Z=0.008)$,

9 http://stev.oapd.inaf.it/cgi-bin/cmd
Table 4. Optical brightness of the point-like counterpart of NGC $5585 \mathrm{X}-1$, determined from the HST observations.

\begin{tabular}{lccc}
\hline \hline Filter & $\begin{array}{c}\text { Exp. Time } \\
(\mathrm{s})\end{array}$ & $\begin{array}{c}\text { Apparent Brightness } \\
(\mathrm{mag})\end{array}$ & $\begin{array}{c}\text { Absolute Brightness } \\
(\mathrm{mag})\end{array}$ \\
\hline F336W & 1200 & $23.80 \pm 0.04$ & $-5.79 \pm 0.04$ \\
F555W & 1245 & $25.27 \pm 0.03$ & $-4.29 \pm 0.03$ \\
F814W & 1350 & $25.90 \pm 0.10$ & $-3.65 \pm 0.10$ \\
\hline
\end{tabular}

$a$ : corrected for Galactic foreground reddening $E(B-V)=0.014$ mag.

expected for the outskirts of a small disk galaxy, as suggested by the analysis of Ganda et al. (2007) and also confirmed by our subsequent spectral analysis (Section 3.3.7). We found that the optical magnitudes are consistent either with an 08.5-O9 main sequence star, or with a B0 subgiant, with a characteristic temperature $T \approx(30,000 \pm 2,000) \mathrm{K}$. The mass range goes from $M \approx 25 M_{\odot}$ for an $\mathrm{O} 8.5 \mathrm{~V}$ star (age $\lesssim 3 \mathrm{Myr}$ ), to $M \approx 15 M_{\odot}$ for a B0IV star (age $\approx 12 \mathrm{Myr}$ ). Alternatively, we used the diskir model in XsPEC, fitted to both the X-ray and optical datapoints. The two model parameters that constrain the optical/UV flux are the outer disk radius (expressed as the ratio of outer/inner disk radii) and the reprocessing fraction, which tells us how much of the illuminating X-ray flux is intercepted and re-emitted by the disk (mostly in the optical/UV band). We found that an accretion disk with an outer radius of $\sim$ a few $10^{11}-10^{12} \mathrm{~cm}$, and an X-ray reprocessing fraction $f \sim 10^{-2}$ also produces optical emission consistent with the observed colours; such a high reprocessing factor is not unusual in ULXs (Sutton et al. 2014), possibly because outflows enhance the fraction of X-ray photons down-scattered onto the surface of the outer disk. Thus, we cannot distinguish between the star and irradiated disk scenarios. One (weak) argument in favour of a significant disc contribution to the optical counterpart is that this is the only blue optical source within $\approx 100$ pc around $\mathrm{X}-1$; massive stars rarely come in isolation.

\subsection{ULX optical bubble versus H II region}

\subsubsection{Morphology}

From the HST image of the bubble in the F657N band (Figures 2, 4), we estimate a diameter of $\approx 9^{\prime \prime} .0$ in the north-south direction and $\approx 5^{\prime \prime} .7$ in the east-west direction. X-1 is roughly in the centre of the bubble, $\approx 4^{\prime \prime}$ from the northern edge along the major axis, and $\approx 3^{\prime \prime}$ from the eastern edge along the minor axis. At the assumed distance of $8 \mathrm{Mpc}$, the bubble size corresponds to $\approx 350 \times \approx 220 \mathrm{pc}$, and its volume is $\approx 3 \times 10^{62} \mathrm{~cm}^{3}$ for a prolate spheroid approximation (i.e., with radii of 110,110 and $175 \mathrm{pc})$. An essentially identical size $(\approx 350 \times 230 \mathrm{pc})$ is independently estimated from the $1.5-\mathrm{GHz}$ VLA image (Figure 2). It is one of the largest ULX bubbles known to-date, in the same class as the "classical" bubbles around NGC $1313 \mathrm{X}-2$, Holmberg IX X-1 and NGC 7793-S26. This size does not include the additional, smaller photo-ionized nebula at the northern end, energized by a small cluster of young stars.

The narrow-band $H S T$ imaging already visually suggests that the ULX bubble is shock-ionized, from its relatively high brightness in [S II] $\lambda 6716,6731$ (F673N filter) and [Fe II] $\lambda 1.64 \mu \mathrm{m}$ (F164N) (Figure 2); instead, there is no discernible emission from those two lines in the northern photo-ionized region. Radio emission is also associated only with the shock-ionized ULX bubble. 

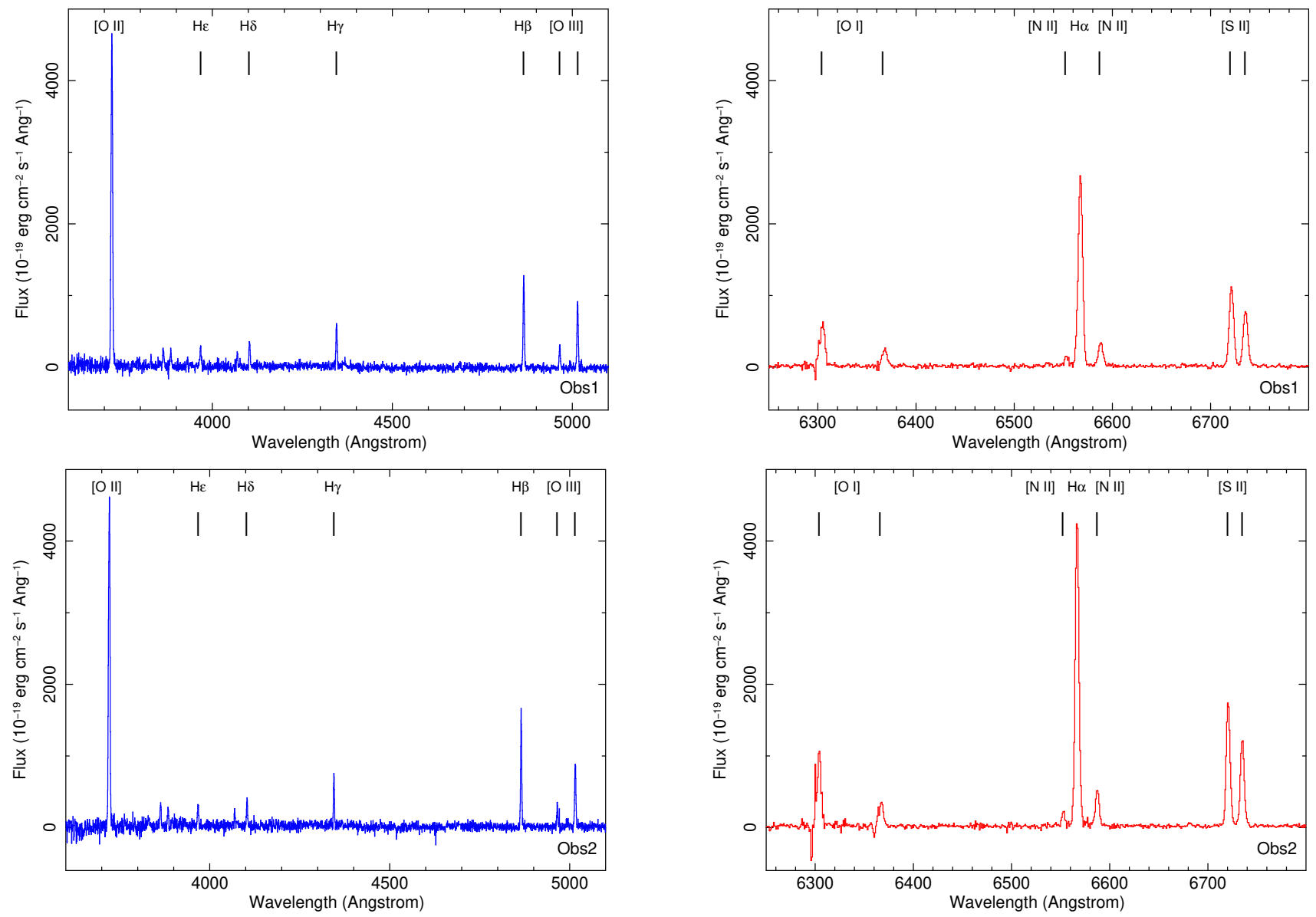

Figure 8. Top panel: blue portion of the LBT spectrum of the ULX bubble (excluding the $\mathrm{H}$ II region) with the slit oriented along the major axis of the bubble (Obs1). Bottom panel: blue spectrum with the slit oriented along the minor axis (Obs2). In both panels, the main lines are identified.

In the rest of this Section 3.3, we will use the LBT spectra (Figures 8,9 ) to calculate intensity ratios between the main diagnostic lines, and determine quantities such as temperature, density and metal abundance of the emitting gas. We will use the HST imaging data to measure the total flux and luminosity in the main lines.

\subsubsection{Diagnostic line ratios}

The first basic classification of an optical nebula is based on the ratio between the flux in the sulphur doublet [S II] $\lambda \lambda 6716,6731$ and in $\mathrm{H} \alpha$ (Mathewson \& Clarke 1973; Blair \& Long 1997). High values of this ratio $(\gtrsim 0.3)$ imply collisional ionization. Because of the small wavelength difference between the lines, the differential reddening is negligible and the ratio is well estimated directly from the observed flux values. We measured the flux ratio along the slit in the both the Obs1 (major axis of the nebula, roughly north to south) and Obs2 (minor axis, roughly east to west) positions. For Obs1, we obtained a $[\mathrm{S} \mathrm{II}] / \mathrm{H} \alpha$ ratio of $\approx 0.72$ (defining the shockionized ULX bubble) between $\approx 4^{\prime \prime} .5$ to the south and $\approx 3^{\prime \prime}$ to the north of the ULX position, measured along the slit (Table 5 and top panel of Figure 10). Further to the north direction, the flux ratio drops rapidly, reaching an average value of $\approx 0.14$ for slit positions between $\approx 4^{\prime \prime} .5$ and $6^{\prime \prime} .2$ from the ULX; this is the location of the photo-ionized H II region. In the Obs2 position, the slit intercepts

Figure 9. Top panel: red portion of the LBT spectrum with the slit oriented along the major axis of the bubble (Obs1), excluding the $\mathrm{H} \mathrm{II} \mathrm{region.} \mathrm{Bottom}$ panel: red spectrum with the slit oriented along the minor axis (Obs2). In both panels, the main lines are identified.

only the ULX bubble; we measure an average flux ratio of $\approx 0.71$ (Table 5).

The second important line ratio considered in our analysis is [S II] $\lambda 6716 / 6731$, an indicator of electron density (Osterbrock $\&$ Ferland 2006). We measured (Table 5) a ratio of $\approx 1.47$ in the bubble region of Obs $1, \approx 1.44$ for Obs 2 , and $\approx 1.5$ for the $\mathrm{H}$ II region of Obs1. All three values fall in the asymptotic low-density regime, $n_{e} \lesssim$ a few $\times 10 \mathrm{~cm}^{-3}$. We will show later (Section 4.1) that the density of the ambient (non-shocked) interstellar medium is $\sim 1$ $\mathrm{cm}^{-3}$. Standard magnetohydrodynamic relations show that for a radiative shock in the presence of magnetic fields, the compression factor (density ratio) across the shock is $\rho_{2} / \rho_{1} \approx b \mathscr{M}^{2}$, where $\mathscr{M}$ is the upstream isothermal Mach number; the exact value of the proportionality constant $b$ depends on the small-scale properties of turbulence and magnetic field, but it is always $b<1(b \approx 1$ in the non-magnetic case). For example, for a magnetic field scaling as $B \propto \rho^{1 / 2}$, the compression factor $\rho_{2} / \rho_{1} \approx 0.16[\beta /(\beta+1)] \mathscr{M}^{2}$ (Molina et al. 2012; Federrath et al. 2010), where $\beta$ is the ratio of gas pressure over magnetic pressure in the pre-shock region. In the case of the X-1 bubble, we will see later (Section 3.3.3) that for a typical sound speed $\sim 10 \mathrm{~km} \mathrm{~s}^{-1}$ in the ambient medium, the Mach number of the shock is $\sim 10-15$. Therefore, we expect a post-shock compression factor of $\sim(1$ to a few $) \times 10$ (that is, $n_{e} \lesssim$ a few $\times 10$ $\mathrm{cm}^{-3}$ ). This is indeed consistent with the density measured from the [S II] $\lambda 6716 / 6731$ doublet. 
Thirdly, we measured the index $R \equiv$ $([\mathrm{OIII}] \lambda 4959+[\mathrm{OIII}] \lambda 5007) /[\mathrm{OIII}] \lambda 4363$. This is a standard indicator of the electron temperature $T_{e}$ (Osterbrock \& Ferland 2006). Nebulae ionized by stellar continua and/or by $\mathrm{X}$-ray emission have typical $T_{e} \approx 10,000 \mathrm{~K}$, while a temperature $T_{e} \gtrsim 20,000 \mathrm{~K}$ typically identifies collisionally ionized gas. In our case, we find: $R \approx 16 \pm 2$ for the ULX bubble in Obs1, which corresponds to $T_{e} \approx(45,000 \pm 3000) \mathrm{K} ; R \approx 18 \pm 2$ for the ULX bubble in Obs2, which corresponds to $T_{e} \approx(42,000 \pm 3000)$ $\mathrm{K}$; and $R \approx 240 \pm 60$ for the $\mathrm{H}$ II region, which corresponds to $T_{e} \approx(9700 \pm 600) \mathrm{K}$. This is consistent with our interpretation of the two regions.

The intensity ratio between $[\mathrm{O} \mathrm{III}] \lambda 5007$ and $\mathrm{H} \beta$ shows (Figure 10 , bottom panel) peaks at the edge of the ULX bubble and in the $\mathrm{H}$ II region, but has its lowest value inside the bubble, close to the ULX position. X-ray photo-ionization by a central source, on the other hand, would result in higher excitation, i.e., higher [O $\mathrm{III}] \lambda 5007 / \mathrm{H} \beta$ ratios, close to the ionizing source. This is indeed observed in several other ULX bubbles, suggesting that both shockand photo-ionisation play a role in those objects. However, for the NGC 5585 X-1 bubble, shock-excitation appears to be by far the dominant mechanism.

Another useful intensity ratio often used in the literature is [O II] $\lambda \lambda 3727,3729$ over [O III] $\lambda 5007$. This ratio often suffers more from uncertainties in the intrinsic extinction; however, it provides a clean distinction between X-ray photoionization and collisional ionization. Specifically, values of $I(3727,3729) / I(5007) \sim 1-$ 10 indicate shocks, while $I(3727,3729) / I(5007)<1$ are exclusive of a power-law ionizing flux (Baldwin et al. 1981; Moy \& Rocca-Volmerange 2002). In this ULX bubble, we measure $I(3727,3729) / I(5007) \approx 6.7(\approx 7$ after de-reddening $)$, near the upper range of the shock-ionization regime.

A list of the observed intensities of the most important lines significantly detected in the LBT spectra is reported in Table 5, expressed as a ratio to the $\mathrm{H} \beta$ intensity $(=100)$; they are divided into the three spatially distinct components described earlier (collisionally ionized ULX bubble along the minor axis and along the major axis, and photo-ionized $\mathrm{H}$ II region). We also list in Table 5 the average intensity of the $\mathrm{H} \alpha$ and $\mathrm{H} \beta$ emission measured along the slit, in units of erg cm$~_{-2} \mathrm{~s}^{-1} \operatorname{arcsec}^{-2}$. The total fluxes and luminosities of those two lines (and of the other lines, via their line ratios) can be estimated from those values, multiplied by the projected sky area of the bubble and of the $\mathrm{H}$ II region. We verified that those estimates are consistent with the direct measurements of narrow-band fluxes from the HST images (Section 3.3.6), and with the values measured for this bubble by Matonick \& Fesen (1997).

\subsubsection{Shock velocity from optical line width}

Intrinsic broadening of the emission lines is another strong clue that a nebula is shock-ionized rather than photo-ionized; this is for example a classic selection criterion between SNRs and H II regions (Points et al. 2019). In our LBT study, we used the intrinsic full-width-half-maximum $\left(\mathrm{FWHM}_{\mathrm{int}}\right)$ of the emission lines from collisionally ionized plasma as a proxy for the shock velocity $v_{\mathrm{s}}$. Even for the simple case of a spherical bubble expanding in a uniform medium, the relation between these two quantities depends on whether the shocks are adiabatic or fully radiative, and on what portion of the bubble is observed. We discuss and quantify this relation in Appendix A; the take-away message of this analysis is that $v_{\mathrm{S}}$ is expected to be $\sim 0.5$ times the FWHM measured from
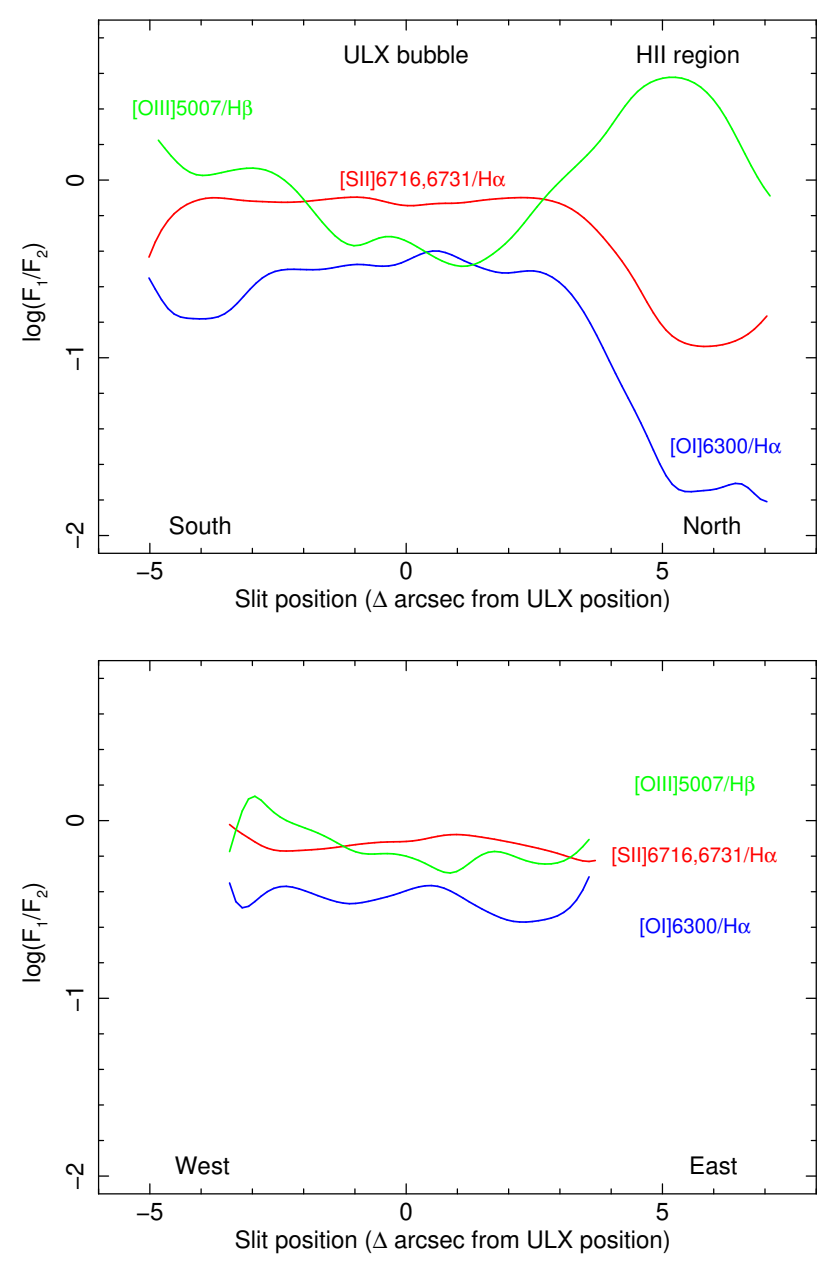

Figure 10. Top panel: diagnostic line ratios $[\mathrm{S}$ II $] / \mathrm{H} \alpha$ (red), $[\mathrm{O} \mathrm{I}] / \mathrm{H} \alpha$ (blue) and $[\mathrm{O} \mathrm{III}] / \mathrm{H} \beta$ (green), plotted along the slit in the Obs1 position (i.e., along the major axis of the bubble, from south to north; see also Figure 4). All curves were smoothed using a Gaussian kernel with a 3-pixel sigma $\left(\approx 0^{\prime \prime} .4\right)$. The drop in the flux ratios $[\mathrm{S} \mathrm{II}] / \mathrm{H} \alpha$ and $[\mathrm{O} \mathrm{I}] / \mathrm{H} \alpha$, and the corresponding increase in $[\mathrm{O} \mathrm{III}] / \mathrm{H} \beta$, mark the transition between shockionized ULX bubble and photo-ionized H II region, $\approx 4$ " north of X-1 (but partially overlapping). Bottom panel: as in the top panel, but along the slit in the Obs2 position, from west to east.

the central region of the bubble, and $\sim 3 / 4$ of the average FWHM integrated over the whole bubble.

To obtain the FWHM $_{\text {int }}$ of the main lines from the bubble region, we need to subtract the instrumental width $\mathrm{FWHM}_{\text {ins }}$ from the observed width $\mathrm{FWHM}_{\mathrm{obs}}$, using the relation $\mathrm{FWHM}_{\mathrm{int}}=$ $\left(\mathrm{FWHM}_{\mathrm{obs}}^{2}-\mathrm{FWHM}_{\mathrm{ins}}^{2}\right)^{1 / 2}$. We used the narrow emission lines from the photo-ionized [H II] region north of the ULX bubble to determine the instrumental line width in our blue and red LBT spectra, separately for each night. For the blue spectra, we found an instrumental width $\mathrm{FWHM}_{\text {ins, } \mathrm{b}} \approx 2.05 \AA$ on the first night and $\mathrm{FWHM}_{\mathrm{ins}, \mathrm{b}} \approx 2.13 \AA$ on the second night; for the red spectra, we found $\mathrm{FWHM}_{\mathrm{ins}, \mathrm{r}} \approx 3.40 \AA$ and $\mathrm{FWHM}_{\mathrm{ins}, \mathrm{r}} \approx 3.41 \AA$ on the two nights. We then measured the $\mathrm{FWHM}_{\mathrm{obs}}$ of $\mathrm{H} \gamma, \mathrm{H} \beta$ and [O III] $\lambda 5007$ in the blue spectra, and of $\mathrm{H} \alpha$, [S II] 66716 and [S II] $\lambda 6731$ in the red spectra, in order to determine their $\mathrm{FWHM}_{\text {int }}$. We converted the width of each line into velocity units and computed an average values between those six lines.

More specifically, we measured the $\mathrm{FWHM}_{\mathrm{obs}}$ of those strong 

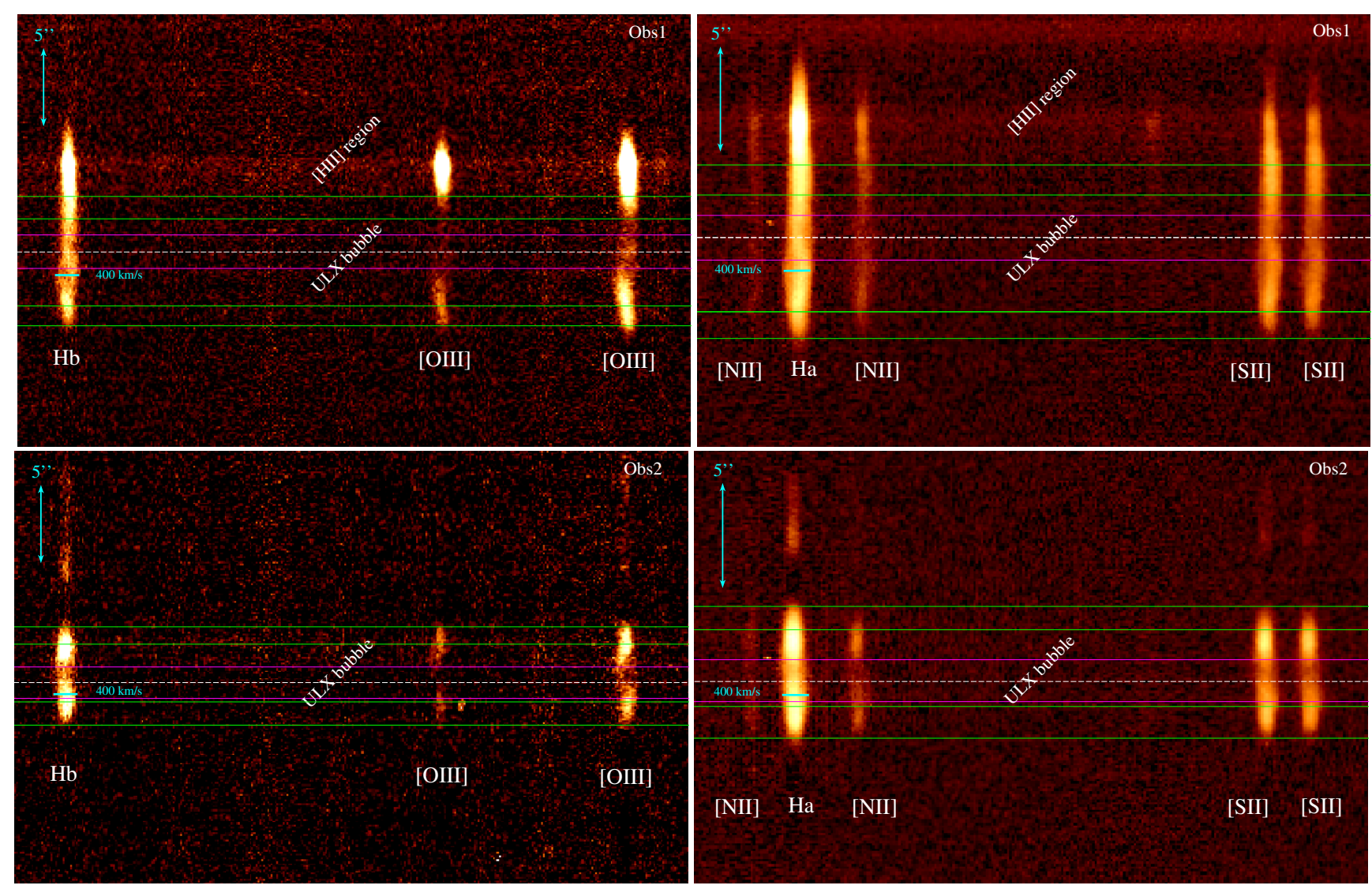

[NII] $\mathrm{Ha} \quad[\mathrm{NII}$

[SII] [SII]

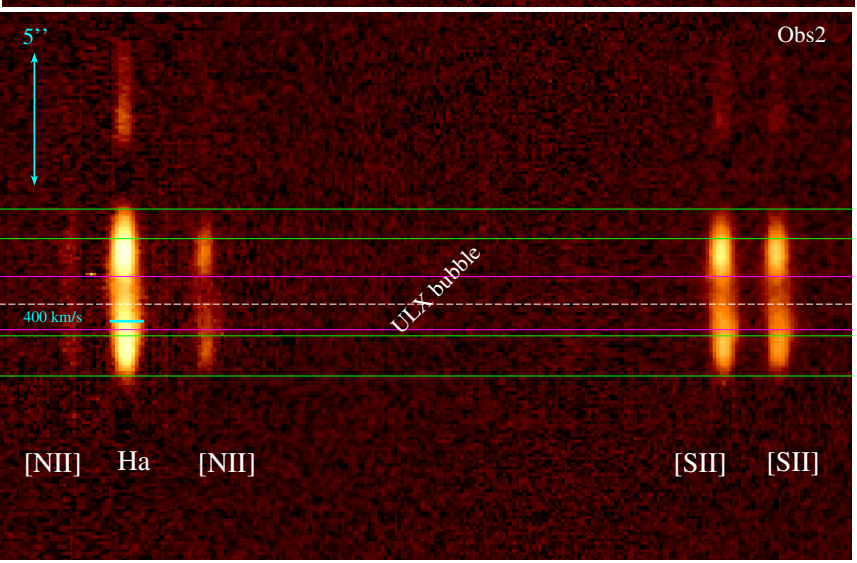

Figure 11. Sky-subtracted, 2-dimensional LBT spectra in the Obs1 (top panels) and Obs2 (bottom panels) slit positions. In the top panels, north is up; in the bottom panels, east is up. The left panels illustrate the region between $\mathrm{H} \beta$ and [O III] $\lambda 5007$; the right panels are for the region between $\mathrm{H} \alpha$ and [S II] $\lambda 6731$ (notice the different zoom factor of left and right panels, for graphical purposes only). Parallel magenta lines define the "central" location of the bubble, defined as $\pm 1^{\prime \prime}$ either side of the location of X-1 along both sits; this is the central region used in Section 3.3.3 to estimate $v_{\mathrm{s}}$. The dashed white line is the location of X-1. A $400 \mathrm{~km} \mathrm{~s}^{-1}$ horizontal bar is overplotted to highlight the observed line broadening. Parallel solid green lines mark four spectral regions at the four edges of the bubble (defined in Section 3.3.3), which we chose for further spatially resolved analysis of FWHMs and Doppler shifts of the lines (Sections 3.3.3, 3.3.4). Notice also that in the Obs1 position, the H II region is well visible (and partly overlapping) at the top end of the ULX bubble, while of course it is not on the slit in the Obs2 position.

lines separately in two ways: i) from the central section of the ULX bubble in both slits (the section of the 2-dimensional spectra between the parallel magenta lines overplotted in Figure 11, defined as $\pm 1^{\prime \prime}$ around the ULX position); ii) integrated over the whole ULX bubble region in both slits. For the central region, after correcting for the instrumental broadening, and averaging over the two slits and the two nights of observations, we find an average FWHM $_{\text {int,c }} \approx$ $210 \pm 20 \mathrm{~km} \mathrm{~s}^{-1}$. From the relations discussed in Appendix A, this suggests $v_{\mathrm{S}} \sim 100 \pm 10 \mathrm{~km} \mathrm{~s}^{-1}$. For the full slit spectra (which is a proxy for the spectrum of the full bubble), we find instead an average $\mathrm{FWHM}_{\mathrm{int}, \mathrm{T}} \approx 170 \pm 30 \mathrm{~km} \mathrm{~s}^{-1}$, which corresponds to $v_{\mathrm{S}} \sim 125 \pm 20 \mathrm{~km} \mathrm{~s}^{-1}$ for a fully radiative shock. We conclude that the most likely value of $v_{\mathrm{S}}$ is between $\sim 100-125 \mathrm{~km} \mathrm{~s}^{-1}$. Henceforth, for the purpose of energy budget and characteristic mechanical power of the bubble, we will take a characteristic $v_{\mathrm{s}} \approx 125 \mathrm{~km} \mathrm{~s}^{-1}$, but our main conclusions would remain essentially valid also for $v_{\mathrm{s}} \approx 100$ $\mathrm{km} \mathrm{s}^{-1}$.

Finally, we extracted spatially resolved spectra also at the outer edges of the bubble region in the two slits (between the parallel green lines overplotted in Figure 11), to measure the line broadening near the northern, southern, eastern and western edges. We defined the four edge regions as follow: along the Obs 1 slit, between $\approx-4^{\prime \prime} .8$ and $-3^{\prime \prime} .5$ to the south, and between $\approx+2^{\prime \prime} .0$ and $+3^{\prime \prime} .4$ to the north of X-1; along the Obs 2 slit, between $\approx-2^{\prime \prime} .7$ and $-1^{\prime \prime} .2$ to the west, and between $\approx+2^{\prime \prime} .5$ and $+3^{\prime \prime} .5$ to the east of X-1. The intensity, velocity and FWHM profiles along the slit of the bright $\mathrm{H} \alpha$ line were used to define and select those edge intervals. Over those regions, the velocity and FWHM of the lines are relatively stable, and therefore we consider them representative of the actual conditions at the expanding front.

We find that the FWHM int $_{\text {varies between }} \approx 110-150 \mathrm{~km} \mathrm{~s}^{-1}$ (averaged over the strongest lines) in the four regions (Figure 12). In principle, the projected velocity exactly at the edge of a radially expanding bubble should be null; however, we are extracting the spectra over finite spatial regions. Moreover, we also see in other bubbles (NGC 7793-S26: M.W. Pakull et al., in prep.) that there is substantial line broadening (of order of the shock velocity) where the outflow impacts the outer shell. This may be evidence of sideways splash or backflow of some of the shocked gas. 


\subsubsection{Systemic velocity and Doppler shifts}

We fitted the strongest lines in the red spectra ${ }^{10}$ with Gaussians, to measure their central positions, and determined an average systemic velocity of $190 \pm 5 \mathrm{~km} \mathrm{~s}^{-1}$ over the whole bubble (average of both slits and both nights), in the heliocentric reference frame. For the $[\mathrm{H} \mathrm{II}]$ region, we measured a heliocentric systemic velocity $\approx 200$ $\pm 10 \mathrm{~km} \mathrm{~s}^{-1}$. The heliocentric velocity of NGC 5585 is $293 \pm 3 \mathrm{~km}$ $\mathrm{s}^{-1}$ (Epinat et al. 2008), but X-1 is located on the approaching side of the rotating galactic disk, where the projected rotational velocity is $\approx-70 \mathrm{~km} \mathrm{~s}^{-1}$ (Côté et al. 1991; Epinat et al. 2008). The fact that $\mathrm{X}-1$ is still located approximately at the centre of its own bubble, after an expansion age of $\approx 6 \times 10^{5} \mathrm{yr}$ (Section 4.1), suggests that the compact object was born with a low kick velocity: we estimate a projected velocity in the plane of the sky $\lesssim 50 \mathrm{~km} \mathrm{~s}^{-1}$.

Furthermore, there are significant velocity differences between different regions of the ULX bubble. From our separate spectral analysis at the four edges of the bubble, we find that the ionized gas at the northern edge is receding at a projected speed $\approx 30 \mathrm{~km} \mathrm{~s}^{-1}$ higher than at the southern edge (Figure 12); conversely, the gas at the eastern edge is moving away $\approx 25 \mathrm{~km} \mathrm{~s}^{-1}$ more slowly than the gas at the western edge and $\approx 30 \mathrm{~km} \mathrm{~s}^{-1}$ more slowly than the gas at the southern edge. In the spectra from the central region, the average recession velocity is $195 \pm 5 \mathrm{~km} \mathrm{~s}^{-1}$.

\subsubsection{Reddening}

We examined the Balmer decrements to obtain a more accurate estimate of the total reddening (Milky Way plus NGC 5585 halo) in front of the bubble and of the H II region. First, we considered the bubble region. The theoretical intensity ratios (without reddening) for collisionally-ionized gas depend only weakly on the shock velocity and metal abundance. Assuming $100 \lesssim v_{\mathrm{S}} \lesssim 150 \mathrm{~km}$ $\mathrm{s}^{-1}$ (Section 3.3.3) and sub-solar (Large Magellanic Cloud) metal abundance (justified by our subsequent analysis in Section 3.3.7 and by the general properties of NGC 5585), from the MAPPINGS III tables of Allen et al. (2008), we expect: $I(\mathrm{H} \alpha) / I(\mathrm{H} \beta) \approx 3.04$ 3.22 (with a central value of $I(\mathrm{H} \alpha) / I(\mathrm{H} \beta) \approx 3.14$ for $v_{\mathrm{s}}=125$ $\left.\mathrm{km} \mathrm{s}^{-1}\right) ; I(\mathrm{H} \gamma) / I(\mathrm{H} \beta) \approx 0.45-0.46 ; I(\mathrm{H} \delta) / I(\mathrm{H} \beta) \approx 0.25$. The observed Balmer flux ratios are: $F(\mathrm{H} \alpha) / F(\mathrm{H} \beta)=3.42 \pm 0.24$; $F(\mathrm{H} \gamma) / F(\mathrm{H} \beta)=0.46 \pm 0.03 ; F(\mathrm{H} \delta) / F(\mathrm{H} \beta)=0.27 \pm 0.03$ (Table 5 , from the average of the Obs 1 and Obs 2 bubble-region values). For the differential reddening between the lines, we used the extinction curve parameterized by Esteban et al. (2014). From $F(\mathrm{H} \alpha) / F(\mathrm{H} \beta)$ we obtain $E(B-V)_{\alpha / \beta}=0.09 \pm 0.08 \mathrm{mag}, E(B-V)_{\gamma / \beta} \lesssim 0.07$ mag, $E(B-V)_{\delta / \beta}<0.08$ mag. Taking an average of the three measurements, we estimate an intrinsic $E(B-V)=0.02_{-0.02}^{+0.04} \mathrm{mag}$, in addition to the Galactic foreground component $E(B-V) \approx 0.014$ mag. Given the small value, low significance and large relative uncertainty of the intrinsic component, in the rest of this paper we will generally correct only for the foreground Galactic extinction, unless explicitly mentioned.

We then used the same Balmer-decrement method to estimate the reddening in the $\mathrm{H}$ II region. Here we assumed Case-B recombination, $T_{e}=10,000 \mathrm{~K}$, low-density limit (Osterbrock \& Ferland 2006); the theoretical flux ratios for photo-ionized gas are: $I(\mathrm{H} \alpha) / I(\mathrm{H} \beta)=2.87 ; I(\mathrm{H} \gamma) / I(\mathrm{H} \beta)=0.468 ; I(\mathrm{H} \delta) / I(\mathrm{H} \beta)=$

10 We only used $\mathrm{H} \alpha$ and the [S II] doublet for the redshift analysis, because the wavelength calibration of the blue spectra has a larger uncertainty, due to the lack of suitable arc lines in that region.

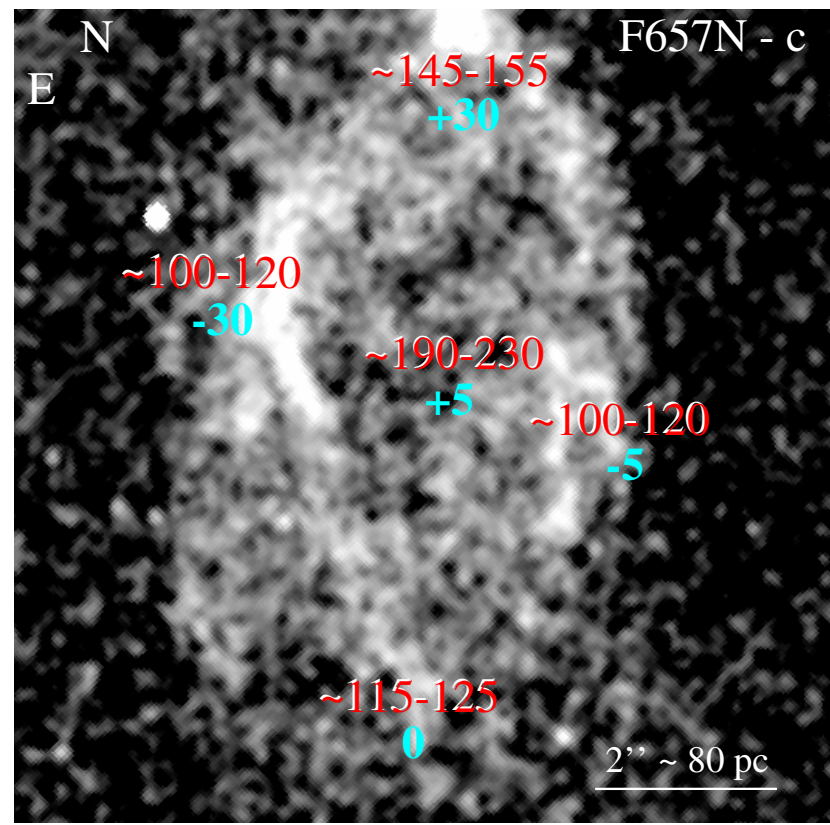

Figure 12. Approximate velocity parameters from an average of the strongest emission lines, superposed on a continuum-subtracted, Gaussiansmoothed image of the ULX bubble in the F657N filter. Red numbers represent the approximate range of intrinsic FWHMs (in $\mathrm{km} \mathrm{s}^{-1}$ ) measured in five characteristic sections of the bubble, from Gaussian fits to the LBT profiles of $\mathrm{H} \alpha, \mathrm{H} \beta, \mathrm{H} \gamma$, [O III] $\lambda 5007$, [S II] $\lambda 6716$ and [S II] 6731 . Blue numbers represent the approximate Doppler shift of the emission lines in the same four regions, with respect to the average systemic velocity over the ULX bubble region. The Doppler shifts are measured from the average shift of the central positions of $\mathrm{H} \alpha$ and [S II] $\lambda 6716$ only; the reference average systemic velocity is $190 \pm 5 \mathrm{~km} \mathrm{~s}^{-1}$.

0.259. In our Obs 1 spectrum, we chose $\mathrm{H} \alpha$ and $\mathrm{H} \delta$ for an estimate of the Balmer decrement (the measured value of $\mathrm{H} \gamma$ is an outlier). Comparing the observed (reddened) values of $F(\mathrm{H} \alpha) / F(\mathrm{H} \beta) \approx 3.9$ and $F(\mathrm{H} \delta) / F(\mathrm{H} \beta) \approx 0.21$ (Table 5) with the theoretical ratios, we obtain $E(B-V)_{\alpha / \beta} \approx 0.30 \mathrm{mag}$ and $E(B-V)_{\delta / \beta} \approx 0.36$ mag. Taking an average of those two values, we obtain a best estimate of the total reddening $E(B-V) \approx 0.33 \mathrm{mag}$.

\subsubsection{Fluxes and luminosities}

The HST image in the F657N band (Table 6) provides an accurate total flux and luminosity for the combined emission of $\mathrm{H} \alpha$, [N II] $\lambda 6584$ and [N II] $\lambda 6548$ in the ULX bubble region. We combined that with the line ratios inferred from the LBT spectra (Table 5), and determined the emitted luminosity of $\mathrm{H} \alpha$ and other lines. We obtain $L_{\mathrm{H} \alpha}=(9.7 \pm 0.7) \times 10^{37} \mathrm{erg} \mathrm{s}^{-1}$ and hence (from the theoretical line ratios) $L_{\mathrm{H} \beta}=(3.2 \pm 0.2) \times 10^{37} \mathrm{erg} \mathrm{s}^{-1}$, where we have corrected only for the foreground Galactic reddening $E(B-V)=$ $0.014 \mathrm{mag}$. If we apply also a (speculative) intrinsic reddening $E(B-V) \approx 0.02 \mathrm{mag}$, the emitted Balmer line luminosity increases to $L_{\mathrm{H} \alpha} \approx 10.2 \times 10^{37} \mathrm{erg} \mathrm{s}^{-1}$ and $L_{\mathrm{H} \beta} \approx 3.4 \times 10^{37} \mathrm{erg} \mathrm{s}^{-1}$. Another important line luminosity that we will use as a proxy for the kinetic energy is the one from [Fe II] $\lambda 1.64 \mu \mathrm{m}$ (F164N filter), for which we infer $L_{[\mathrm{FeII}]}=(1.1 \pm 0.1) \times 10^{37} \mathrm{erg} \mathrm{s}^{-1}$ (Table 6); the additional intrinsic reddening would only increase this value by $\approx 1 \%$ ).

A detailed analysis of the line and continuum emission from the $\mathrm{H}$ II region is beyond the scope of this work. Here we report only a few results that may help characterize the environment around the 
ULX. We determined a total luminosity (corrected for foreground Galactic extinction) of $\approx 3.6 \times 10^{37} \mathrm{erg} \mathrm{s}^{-1}$ in the F657N filter, and we estimate that $\mathrm{H} \alpha$ contributes $\approx 95 \%$ of this emission (i.e., $\approx 3.4$ $\times 10^{37} \mathrm{erg} \mathrm{s}^{-1}$ ). Correcting for the additional intrinsic extinction (Section 3.3.4) $A_{\mathrm{H} \alpha} \approx 0.818 \times 3.1 \times 0.31 \mathrm{mag}$, we infer an emitted luminosity $L_{\mathrm{H} \alpha} \approx 7 \times 10^{37} \mathrm{erg} \mathrm{s}^{-1}$. The intrinsic reddening derived from the Balmer decrement is certainly an upper limit for the reddening of the continuum emission; the latter is dominated by bright stars that may have already cleared the gas around them. The continuum luminosity in the broad-band HST filters is summarized in Table 7.

We ran simulations with the STARBURST 99 web-based software (Leitherer et al. 1999, 2014) to estimate the stellar population properties consistent with such luminosities and colours. We find that both the $\mathrm{H} \alpha$ luminosity and the optical colours are consistent with an instantaneous burst of star formation with a mass $M \approx(2000 \pm 500) M_{\odot}$, after an age $\lesssim 3.5 \mathrm{Myr}$ (the timescale after which $\mathrm{O}$ stars die, which makes the U-band and $\mathrm{H} \alpha$ luminosities drop quickly). Alternatively, we find acceptable solutions for continuous star formation at a rate of $\approx 4-7 \times 10^{-4} M_{\odot} \mathrm{yr}^{-1}$ at an age $\lesssim 4 \mathrm{Myr}$.

\subsubsection{Metal abundance}

We determined the metal abundance independently in the $\mathrm{H}$ II region and in the ULX bubble. For the H II region, first we re-visited our temperature estimate (Section 3.3.3), now taking into account also the reddening derived in Section 3.3.4. We obtain an electron temperature in the [O III] zone $T_{e}(\mathrm{OIII})=10,600 \pm 400 \mathrm{~K}$. The corresponding temperature in the [O II] zone, $T_{e}(\mathrm{OII})$ can be empirically obtained from the scaling relation of Garnett (1992), which gives $T_{e}(\mathrm{OII})=10,400 \pm 400 \mathrm{~K}$. With these two temperatures, and with the reddening-corrected intensity of the strong oxygen lines, we can then determine the oxygen abundance, using the relations of Izotov et al. (2006). We obtain a metallicity in$\operatorname{dex} 12+\log (\mathrm{O} / \mathrm{H}) \approx 8.19$ dex. Using instead the calibration of Pagel et al. (1992) (see also Pilyugin \& Thuan 2005), we obtain $12+\log (\mathrm{O} / \mathrm{H}) \approx 8.17$ dex. Thus, the metal abundance appears to be intermediate between those of the Large Magellanic Cloud $(12+\log (\mathrm{O} / \mathrm{H})=8.35 \pm 0.06)$ and the Small Magellanic Cloud $(12+\log (\mathrm{O} / \mathrm{H})=8.03 \pm 0.10)$ (Russell \& Dopita 1992). An alternative calibration for the oxygen abundance in an $\mathrm{H}$ II region is based on the intensity ratio [O II] $\lambda 6583 / \mathrm{H} \alpha$ (Pérez-Montero \& Contini 2009). In our case, we measured [O II] $] 6583 / \mathrm{H} \alpha \approx 0.05$, which implies an abundance $12+\log (\mathrm{O} / \mathrm{H}) \approx 8.04$, consistent with the Small Magellanic Cloud.

For the ULX bubble, we used again the ratio [N II] $] 6583 / \mathrm{H} \alpha$, which is a strong function of metallicity in shock-ionized gas (Allen et al. 2008). The empirical ratio in the bubble region is $\approx 0.14 \pm 0.02$ (Table 5 ), which is similar to the ratios $(\approx 0.10-0.11)$ predicted by the MAPPINGS III code for shock velocities in the range $\approx 100-150$ $\mathrm{km} \mathrm{s}^{-1}$ at Large Magellanic Cloud metallicity. As a comparison, the same line ratio is predicted to be $\approx 0.04-0.05$ at Small Magellanic Cloud metallicity, and $\approx 0.48-0.51$ at solar metallicity. Thus, it is possible that the more evolved bubble region is also slightly more enriched than the currently star-forming region north of it, but they are both sub-solar.

\subsubsection{Another diagnostic line: [O I] $\lambda 6300$}

The low-ionization line [O I] $\lambda 6300$ is another indicator of the spatial boundary between the collisionally ionized ULX bubble and
Table 5. Observed relative intensity ${ }^{a}$ of the main lines in the ULX bubble and $\mathrm{H}$ II regions, from the LBT spectra, and absolute average intensity of $\mathrm{H} \beta$. Errors are $\sim 10 \%$ for the weaker lines (those with an intensity $\lesssim 0.5$ times $\mathrm{H} \beta$ ), and $\sim 5 \%$ for the stronger lines (those with an intensity $\gtrsim \mathrm{H} \beta$ ).

\begin{tabular}{|c|c|c|c|}
\hline \multirow[t]{2}{*}{ Line } & \multicolumn{3}{|c|}{ Extraction Region } \\
\hline & $\begin{array}{c}\text { ULX bubble }^{b} \\
\text { Obs1 slit }\end{array}$ & $\begin{array}{c}\text { ULX bubble }^{c} \\
\text { Obs2 slit }\end{array}$ & $\begin{array}{c}\text { H II region }{ }^{d} \\
\text { Obs1 slit }\end{array}$ \\
\hline & \multicolumn{3}{|c|}{ Relative Intensities } \\
\hline [O II] $] \lambda \lambda 3727,3729$ & 502 & 469 & 115 \\
\hline$[\mathrm{Ne} \mathrm{III}] \lambda 3869$ & 20 & 26 & 16 \\
\hline $\mathrm{H} \zeta \lambda 3889$ & & & 10 \\
\hline${ }^{e} \mathrm{H} \epsilon \lambda 3970$ & 27 & 24 & 14 \\
\hline [S II] $\lambda 4069$ & 18 & 11 & \\
\hline Н $\delta \lambda 4102$ & 27 & 27 & 21 \\
\hline $\mathrm{H} \gamma \lambda 4340$ & 48 & 43 & 34 \\
\hline [O III] $\lambda 4363$ & 6 & 5 & 2 \\
\hline $\mathrm{H} \beta \lambda 4861$ & 100 & 100 & 100 \\
\hline [O III] $\lambda 4959$ & 20 & 21 & 117 \\
\hline [O III] $\lambda 5007$ & 77 & 69 & 353 \\
\hline He I $\lambda 5876$ & 16 & 10 & 15 \\
\hline [O I] $\lambda 6300$ & 93 & 76 & 7 \\
\hline [S III] $\lambda 6312$ & & & 4 \\
\hline [O I] $\lambda 6364$ & 33 & 23 & 3 \\
\hline [N II] $] 6548$ & 21 & 16 & 3 \\
\hline $\mathrm{H} \alpha \lambda 6563$ & 356 & 328 & 391 \\
\hline [N II] $\lambda 6583$ & 55 & 44 & 19 \\
\hline [S II] $\lambda 6716$ & 153 & 138 & 33 \\
\hline [S II] $\lambda 6731$ & 104 & 96 & 22 \\
\hline
\end{tabular}

Average Intensity $f$

\begin{tabular}{|c|c|}
\hline $\mathrm{H} \beta \lambda 4861$ & $1.0 \pm 0.1$ \\
\hline
\end{tabular}

$a$ : normalized to $\mathrm{H} \beta$, without reddening corrections;

b: ULX bubble spectrum extracted between $-4{ }^{\prime \prime} .3$ and $+2^{\prime \prime} .0$ from the ULX position (positive values meaning to the north of the ULX), along the slit in the Obs 1 position. The ULX bubble extends from $\approx-5^{\prime \prime}$ to $\approx+4^{\prime \prime}$ from the ULX position; however, we only used the slit section between $-4^{\prime \prime} .3$ and $+2^{\prime \prime} .0$ for the line intensities in this Table, to maximize signal-to-noise and avoid contamination from the $\mathrm{H}$ II region.

$c$ : ULX bubble spectrum extracted from Obs2, between $-2^{\prime \prime} .7$ and $+3^{\prime \prime} .5$

from the ULX position (positive values meaning to the east);

$d$ : H II region spectrum extracted between $+4^{\prime \prime} .5$ and $+6^{\prime \prime} .2$ from the ULX position, along the slit in the Obs 1 position;

$e$ : blended with [Ne III] $\lambda 3968$ and Ca II $\lambda 3969$;

$f$ : average intensity on the slit, in units of $10^{-16} \mathrm{erg} \mathrm{cm}^{-2} \mathrm{~s}^{-2} \operatorname{arcsec}^{-2}$.

the photo-ionized H II region (Figure 10, top panel). Surprisingly, the average observed intensity ratio $I(6300) / I(\mathrm{H} \alpha) \approx 0.25$ (Table 5 ) in the ULX bubble is much higher than predicted by the MAPPINGS III tables, which suggest instead values in the range $\approx 0.02-$ 0.05 for a plausible grid of shock velocities $\left(100 \mathrm{~km} \mathrm{~s}^{-1}, 125 \mathrm{~km}\right.$ $\mathrm{s}^{-1}, 150 \mathrm{~km} \mathrm{~s}^{-1}$ ), metallicities (Small Magellanic Cloud, Large Magellanic Cloud, solar) and magnetic fields $(B=0, B=1 \mu \mathrm{G}$, $B=3.23 \mu \mathrm{G}$; the last value represents the field at thermal $/$ magnetic 
Table 6. Continuum-subtracted narrow-band emission from the ULX bubble

\begin{tabular}{lccc}
\hline \hline Filter/Line & $\begin{array}{c}\text { Exp. Time } \\
(\mathrm{s})\end{array}$ & $\begin{array}{c}\text { Flux } \\
\left(\mathrm{erg} \mathrm{cm}^{-2} \mathrm{~s}^{-1}\right)\end{array}$ & $\begin{array}{c}\text { Luminosity }^{a} \\
\left(\mathrm{erg} \mathrm{s}^{-1}\right)\end{array}$ \\
\hline $\mathrm{F}^{2} 02 \mathrm{~N}^{b}$ & 2012 & $(3.3 \pm 0.6) \times 10^{-15}$ & $(2.7 \pm 0.5) \times 10^{37}$ \\
$\mathrm{~F}{ }^{c}$ & 2916 & $(14.4 \pm 1.0) \times 10^{-15}$ & $(11.4 \pm 0.8) \times 10^{37}$ \\
$\mathrm{~F}^{c}$ & $213 \mathrm{~N}^{d}$ & $(8.5 \pm 0.5) \times 10^{-15}$ & $(6.8 \pm 0.4) \times 10^{37}$ \\
$\mathrm{~F} 64 \mathrm{~N}^{e}$ & 1659 & $(1.5 \pm 0.1) \times 10^{-15}$ & $(1.1 \pm 0.1) \times 10^{37}$
\end{tabular}

$a$ : corrected for foreground Galactic reddening $E(B-V)=0.014$ mag;

$b$ : Covering [O III] $\lambda 5007$;

${ }^{c}$ : Covering $\mathrm{H} \alpha$, [N II] $] 26548,6583$. H $\alpha$ contributes $\approx 85 \%$ of the flux and luminosity in this filter, based on the LBT line ratios;

$d$ : Covering [S II] $\lambda 6716,6731$;

$e^{e}$ : Covering $[\mathrm{Fe} \mathrm{II}] \lambda 16,440$.

pressure equipartition, for a gas density $n=1 \mathrm{~cm}^{-3}$ ). Intensity ratios $\gtrsim 0.2$ apparently require shock velocities $\gtrsim 600 \mathrm{~km} \mathrm{~s}^{-1}$ according to the MAPPINGS III code; this is clearly ruled out by the rest of our data. On the other hand, other widely used shock ionization models (Cox \& Raymond 1985; Hartigan et al. 1987, 1994) do predict intensity ratios in our observed range; for example, a ratio $\approx 0.3$ for a shock velocity $\approx 140 \mathrm{~km} \mathrm{~s}^{-1}$ into fully neutral material (Cox \& Raymond 1985). As an observational comparison, values of $I(6300) / I(\mathrm{H} \alpha) \approx 0.25-0.30$ were found across the collisionally ionized Galactic supernova remnant W44, for which the estimated shock velocity is $\approx 110-150 \mathrm{~km} \mathrm{~s}^{-1}$ (Mavromatakis et al. 2003).

\subsection{ULX radio bubble}

Our VLA observations reveal for the first time a bright radio nebula (size of $350 \times 230 \mathrm{pc}$ ) associated with the optical/infrared ULX bubble (Figure 2). We measured an integrated flux density $S_{v}=$ $1.4 \pm 0.1 \mathrm{mJy}$ at $1.5 \mathrm{GHz}$. At the assumed distance of $8 \mathrm{Mpc}$, this corresponds to a 1.5-GHz luminosity density $L_{v}=(1.1 \pm 0.1) \times 10^{26}$ $\mathrm{erg} \mathrm{s}^{-1} \mathrm{~Hz}^{-1}$ and a luminosity $L \equiv v L_{v}=(1.6 \pm 0.1) \times 10^{35} \mathrm{erg}$ $\mathrm{s}^{-1}$. Assuming an optically thin synchrotron spectrum of index $\alpha=-0.7$ (with $S_{v} \propto v^{\alpha}$ ), this corresponds to a 5-GHz luminosity $L=(2.3 \pm 0.2) \times 10^{35} \mathrm{erg} \mathrm{s}^{-1}$. As a comparison, the radio bubble associated with S26 in NGC 7793 (prototypical example of a jetpowered bubble) has a size of $\approx 300 \times 150 \mathrm{pc}$ and a 5 -GHz luminosity $L=(1.6 \pm 0.1) \times 10^{35} \mathrm{erg} \mathrm{s}^{-1}$.

Although synchrotron emission is likely the dominant component, the strong Balmer line emission observed from the bubble suggests that there might also be a contribution to the radio continuum from free-free emission. However, using the ratio of $\mathrm{H} \beta$ and radio emissivities from Caplan \& Deharveng (1986), at a temperature of $\sim 40,000 \mathrm{~K}$, we verified that free-free emission is expected to contribute only $\approx 2 \%$ of the observed radio luminosity.

The radio nebula shows three regions of significantly enhanced emission (Figure 2). Two of them are consistent with knots or hot spots along the major axis (that is, along the putative direction of a jet, by analogy with S26). The third one appears to coincide with the shock front on the eastern side of the bubble, which is also the brightest in the optical/infrared lines, perhaps because the shock is advancing into an ambient medium with higher density.
Table 7. Continuum brightness of the star clusters in the H II region.

\begin{tabular}{lccc}
\hline \hline Filter & $\begin{array}{c}\text { Exp. Time } \\
(\mathrm{s})\end{array}$ & $\begin{array}{c}\text { Apparent Brightness } \\
(\mathrm{mag})\end{array}$ & $\begin{array}{c}\text { Absolute Brightness } \\
(\mathrm{mag})\end{array}$ \\
\hline F336W & 1200 & $20.1 \pm 0.04$ & $-9.5 \pm 0.1$ \\
F555W & 1245 & $21.4 \pm 0.03$ & $-8.2 \pm 0.1$ \\
F814W & 1350 & $21.3 \pm 0.10$ & $-8.3 \pm 0.1$ \\
\hline
\end{tabular}

$a$ : corrected for foreground Galactic reddening $E(B-V)=0.014$ mag.

\section{DISCUSSION}

\subsection{Jet power derived from the ULX bubble}

Following Pakull et al. (2010), we estimate the kinetic power of the bubble in two ways. First, we use a well known relation from standard bubble theory (Weaver et al. 1977), between kinetic power $P_{\mathrm{w}}$, size $R$ and age $t$ of the bubble, and mass density $\rho_{0}$ of the undisturbed interstellar medium:

$R \approx 0.76\left(P_{\mathrm{w}} / \rho_{0}\right)^{1 / 5} t^{3 / 5}$.

We define $R_{2} \equiv R /(100 \mathrm{pc}) ; t_{5} \equiv t /\left(10^{5} \mathrm{yr}\right) ; P_{39} \equiv$ $P_{\mathrm{w}} /\left(10^{39} \mathrm{erg} \mathrm{s}^{-1}\right)$. We also assume the bubble is expanding into mostly neutral ambient gas, for which the mean molecular weight $\mu \approx 1.30$, and $\rho_{0} \equiv \mu m_{\mathrm{p}} n_{0} \approx 2.17 \times 10^{-24} n_{0} \mathrm{~g} \mathrm{~cm}^{-3}$, with $n_{0}$ being the atomic number density of the unshocked medium. Thus, the previous scaling relation can be re-written as

$R_{2} \approx 0.265\left(P_{39} / n_{0}\right)^{1 / 5} t_{5}^{3 / 5}$

with $n_{0}$ in units of $\mathrm{cm}^{-3}$. We adopt an average bubble radius $R \approx 130$ pc, and we estimate the age of the bubble as $t=(3 / 5) R / v_{\mathrm{S}}$ where we identify the expansion speed with the shock velocity (Section 3.3.3). Thus,

$t_{5}=5.9 R_{2} / v_{2} \approx 6.1$

with $v_{2} \equiv v_{\mathrm{s}} /\left(100 \mathrm{~km} \mathrm{~s}^{-1}\right)$ and $v_{\mathrm{s}} \approx 125 \mathrm{~km} \mathrm{~s}^{-1}$. Alternatively, we can take $R$ as the semi-minor axis $\left(R_{2} \approx 1.1\right)$ and $v_{\mathrm{s}} \approx 100 \mathrm{~km} \mathrm{~s}^{-1}$ as estimated for the central region of the bubble: this gives an age $t_{5} \approx 6.5$. Then, Equation (1) transforms to:

$\left(P_{39} / n_{0}\right) \approx 7.7 \times 10^{2} R_{2}^{5} t_{5}^{-3} \approx 13$,

with an uncertainty of a factor of 2 , for a plausible range of shock velocities.

The hydrogen number density $n_{0}$ of the interstellar gas can be estimated from the shock velocity and the radiative flux of the $\mathrm{H} \beta$ line ${ }^{11}$ (Equation 3.4 of Dopita \& Sutherland 1996):

$n_{0} / \mathrm{cm}^{-3}=\left(10^{6} \mathrm{~s}^{3} \mathrm{~g}^{-1}\right) L_{\mathrm{H} \beta} A^{-1}\left(7.44 v_{2}^{2.41}\right)^{-1}$

where the total area $A$ of the spheroidal bubble can be estimated as $A \approx 2.0 \times 10^{42} \mathrm{~cm}^{2}$, and we have already inferred an intrinsic $\mathrm{H} \beta$ luminosity $L_{\mathrm{H} \beta}=(3.2 \pm 0.2) \times 10^{37} \mathrm{erg} \mathrm{s}^{-1}$ (Section 3.3.5). This gives $n_{0}=(1.3 \pm 0.3) \mathrm{cm}^{-3}$, where the error includes the uncertainty in the observed flux (estimated as $\delta f_{\mathrm{H} \beta} / f_{\mathrm{H} \beta} \lesssim 0.1$ ) and in the shock

11 We consider only the main shock contribution to the line luminosity, and not the precursor, because we have already shown (Section 3.3.3) that $v_{\mathrm{S}}<175 \mathrm{~km} \mathrm{~s}^{-1}$. At those relatively low shock velocities, there is no photoionized precursor because the speed of the ionization front becomes lower than $v_{\mathrm{s}}$; see Equation 4.2 in Dopita \& Sutherland (1996). 
velocity $\left(\delta v_{\mathrm{s}} / v_{\mathrm{S}} \lesssim 0.2\right)$. So far, we have not discussed the error on the distance $d$ to NGC 5585. From the standard deviation of redshiftindependent distance measurements listed in the NASA/IPAC Extragalactic Database, we infer $\delta d / d \approx 1.5 / 8$. However, the density scales as $n_{0} \propto f_{\mathrm{H} \beta} d^{2} \Omega^{-1} d^{-2} v_{2}^{-2.41} \propto f_{\mathrm{H} \beta} \Omega^{-1} v_{2}^{-2.41}$, where $\Omega$ is the solid angle of the bubble (an observable quantity); that is, $n_{0}$ is not directly a function of $d$. For plausible errors $\delta \Omega / \Omega \sim 0.1$, Gaussian propagation shows that the relative error $\delta n_{0} / n_{0}$ is dominated by the error on $v_{\mathrm{s}}$. We estimate $n_{0}=(1.3 \pm 0.6) \mathrm{cm}^{-3}$.

The mechanical power is then $P_{\mathrm{w}} \approx 1.6 \times 10^{40} \mathrm{erg} \mathrm{s}^{-1}$, from Equation (4). If we adopt instead an intrinsic $\mathrm{H} \beta$ luminosity $L_{\mathrm{H} \beta} \approx$ $3.4 \times 10^{37} \mathrm{erg} \mathrm{s}^{-1}$ (corresponding to an intrinsic reddening $E(B-$ $V) \approx 0.02 \mathrm{mag}$ ), the inferred mechanical power increases by the same factor, that is $P_{\mathrm{w}} \approx 1.7 \times 10^{40} \mathrm{erg} \mathrm{s}^{-1}$. We can estimate the error on $P_{\mathrm{W}}$ using similar arguments to those used for the error on $n_{0}$. In this case, $P_{\mathrm{w}} \propto n_{0} R^{5} t^{-3} \propto f_{\mathrm{H} \beta} d^{2} v_{\mathrm{s}}^{-2.41} R^{3}\left(R^{-3} v_{\mathrm{s}}^{3}\right) \propto$ $f_{\mathrm{H} \beta} d^{2} v_{\mathrm{s}}^{0.59}$, and we obtain $\delta P_{\mathrm{w}} / P_{\mathrm{w}} \sim 0.4$ within the framework of the assumed model. We conservatively say that our model estimate of $P_{\mathrm{w}}$ is valid within a factor of 2 .

The second method we used to estimate the jet power is based on the luminosity of suitable diagnostic lines, which carry a known fraction of the total kinetic power (Pakull et al. 2010). In particular, the fractional power radiated via $\mathrm{H} \beta$ and $\mathrm{Fe}$ II $\lambda 1.644 \mu$ m depends only weakly on the density and shock velocity across our plausible range of parameters. From Dopita \& Sutherland (1996) and the MAPPINGS III tables of Allen et al. (2008), for Large Magellanic Cloud metallicity (Section 3.3.7) and equipartition magnetic field, we infer that $P_{\mathrm{w}} \approx 1 /\left(1.4 \times 10^{-3}\right) \times L_{\mathrm{H} \beta} \approx 2.3 \times 10^{40} \mathrm{erg} \mathrm{s}^{-1}$ (independent of ambient density), in agreement with the previous estimate of the mechanical power. Varying the shock velocity between $100-150 \mathrm{~km} \mathrm{~s}^{-1}$ leads to an error range of $\approx 20 \%$ around the central estimate; choosing a MAPPINGS III model without magnetic field leads to an increase in the predicted flux by $\approx 12 \%$. Correcting the $\mathrm{H} \beta$ luminosity for an intrinsic reddening $E(B-V) \approx 0.02 \mathrm{mag}$ gives $P_{\mathrm{w}} \approx 2.4 \times 10^{40} \mathrm{erg} \mathrm{s}^{-1}$. If we use the measured Fe II luminosity, for the same choice of MAPPINGS III parameters, we obtain instead $P_{\mathrm{w}} \sim 1 /\left(3.4 \times 10^{-4}\right) \times L_{[\mathrm{FeII}]} \approx 3.2 \times 10^{40} \mathrm{erg} \mathrm{s}^{-1}$, with an uncertainty of $\approx 40 \%$ for the plausible range of shock velocity, and a factor of 2 higher for null magnetic field.

\subsection{Radio luminosity of ULX bubbles}

The integrated radio luminosity is not a particularly reliable proxy for the kinetic power, neither in ULX bubbles nor in radio galaxies, even when the age of the bubble is known. The same kinetic power can produce very different radio luminosities, depending on unobserved quantities such as the filling factor of the magnetic field in the bubble, the minimum and maximum energy of the relativistic electrons, the relative fraction of power carried by protons. Empirical relations between the optically thin synchrotron luminosity of radio lobes and the jet power were proposed for example by Willott et al. (1999), Cavagnolo et al. (2010), and Godfrey \& Shabala (2013), based on samples of radio galaxies. It would be tempting to extrapolate such relations down to the microquasar regime. However, Godfrey \& Shabala (2016) argued that such scalings are spurious relations caused by the common dependence on distance of both axes, when the calibration sample is observed over a small range of apparent luminosities but a much larger range of cosmic distances. This introduces a form of Malmquist bias in favour of the most luminous and powerful sources at each distance. See also Feigelson
\& Berg (1983) and Elvis et al. (1978) for a discussion of the pitfalls of luminosity-luminosity correlations.

Instead of comparing the size and radio luminosity of this ULX bubble with those of lobes and cavities in radio galaxies, it is perhaps more interesting to compare them with the corresponding quantities in SNRs. An analytical model of the evolution of radio SNRs (Sarbadhicary et al. 2017) predicts $1.4-\mathrm{GHz}$ luminosity density

$L_{1.4} \approx 6.7 \times 10^{24}\left(\frac{\epsilon_{e}}{0.01}\right)\left(\frac{\epsilon_{b}^{u}}{0.01}\right)^{0.8} R_{2}^{3} v_{2}^{3.6} \mathrm{erg} \mathrm{s}^{-1} \mathrm{~Hz}^{-1}$

where $R_{2}$ and $v_{2}$ were defined in Section 4.1, $\epsilon_{e}$ is the fraction of kinetic power transferred to relativistic electrons after the shock, and $\epsilon_{b}^{u}$ is the fraction of energy in the amplified upstream magnetic field. To a first approximation, $\epsilon_{e}$ and $\epsilon_{b}^{u}$ can be treated as constant (White et al. 2019), so that the evolution of the radio luminosity density $L_{1.4}$ depends only on $R(t)$ and $v_{\mathrm{S}}(t)$. Let us assume that Eq.(6) holds both for shocked bubbles created by one initial injection of energy $E$ (SNR case) and for those gradually inflated by accretionpowered outflows with constant kinetic power $P_{\mathrm{W}}$ (ULX bubbles). Then, the main difference between the two cases is that for an SNR, $R \propto\left(E / n_{0}\right)^{1 / 5} t^{2 / 5}$ while for a ULX bubble (as already discussed in Section 4.1) $R \propto\left(P_{\mathrm{w}} / n_{0}\right)^{1 / 5} t^{3 / 5}$ (Pakull et al. 2006).

Inserting the scalings of $R(t)$ and $v_{\mathrm{S}}(t)$ for ULX bubbles (Section 4.1), we can recast Equation (6) in these alternative forms, highlighting their dependence of the mechanical power:

$L_{1.4} \approx 6.2 \times 10^{23}\left(\frac{\epsilon_{e}}{0.01}\right)\left(\frac{\epsilon_{b}^{u}}{0.01}\right)^{0.8}\left(\frac{P_{39}}{n_{0}}\right)^{1.32} t_{5}^{0.36} \mathrm{erg} \mathrm{s}^{-1} \mathrm{~Hz}^{-1}$

$L_{1.4} \approx 1.4 \times 10^{24}\left(\frac{\epsilon_{e}}{0.01}\right)\left(\frac{\epsilon_{b}^{u}}{0.01}\right)^{0.8}\left(\frac{P_{39}}{n_{0}}\right)^{1.2} R_{2}^{0.6} \mathrm{erg} \mathrm{s}^{-1} \mathrm{~Hz}^{-1}$

For the observed properties of the ULX bubble in NGC 5585, the predicted radio luminosity density is $L_{1.4} \sim 4 \times 10^{25} \mathrm{erg} \mathrm{s}^{-1}$ $\mathrm{Hz}^{-1}$, within a factor of 3 of the observed luminosity density (Section 3.4). This is a reasonably good agreement, considering the uncertainty on the $\epsilon_{e}$ and $\epsilon_{b}^{u}$ coefficients and the large scatter in the observed radio luminosity of the SNR population. More generally, the synchrotron model of Sarbadhicary et al. (2017) suggests that a radio luminosity $\sim 10^{35} \mathrm{erg} \mathrm{s}^{-1}$ is a characteristic order-ofmagnitude value for ULXs with kinetic power of $\sim 10^{40} \mathrm{erg} \mathrm{s}^{-1}$ and activity age $\sim$ a few $10^{5}$ yr.

It is useful to compare Equations $(7,8)$ with the corresponding expressions for an SNR bubble, derived and discussed in White et al. (2019). The radio luminosity of an SNR bubble decreases with time, during the Sedov phase, as $L_{1.4} \propto t^{-0.96}$ or $L_{1.4} \propto R^{-2.4}$. Conversely, an active ULX bubble increases its radio luminosity as it expands, scaling as $L_{1.4} \propto t^{0.36}$ or $L_{1.4} \propto R^{0.6}$. Thus, a radio SNR with a standard energy $E=10^{51} \mathrm{erg}$ can also reach luminosities $\sim 10^{35} \mathrm{erg} \mathrm{s}^{-1}$, but only at times $t \lesssim 10^{3} \mathrm{yrs}$ and sizes $\lesssim 10 \mathrm{pc}$; radio bubbles with sizes $\sim 100 \mathrm{pc}$ and ages of a few $10^{5} \mathrm{yr}$ can only be powered by super-critical accretion.

\section{CONCLUSIONS}

We showed that the large ionized nebula at the outskirts of NGC 5585 is one of the cleanest examples of shock-ionized ULX bubbles. Its size $(350 \mathrm{pc} \times 220 \mathrm{pc}$, from $H S T$ narrow-band images) 
puts it in the same class as the bubbles around Holmberg IX X-1 (Pakull et al. 2006; Moon et al. 2011), NGC 7793-S26 (Pakull et al. 2010; Soria et al. 2010), and NGC 1313 X-2 (Pakull \& Mirioni 2002; Weng et al. 2014). There is no direct evidence of hot spots or of a jet (unlike in NGC 7793-S26), but we discovered a very strong radio emission, spatially coincident with the optical bubble, with resolved internal structure. In fact, its radio luminosity is slightly higher than in NGC 7793-S26 (Soria et al. 2010); it is ten times more radio luminous than Holmberg IX X-1 (Berghea et al. 2020) and two orders of magnitude more luminous than NGC $1313 \mathrm{X}-2$ (undetected in the radio).

Using HST images and LBT spectra, we resolved the ionized nebula into a main ULX bubble and a smaller H II region apparently located at its northern end. We cannot determine whether the ULX bubble and the H II region are physically connected or their proximity is due to a projection effect. If physically connected, the other intriguing but for the time being unanswerable question is whether the formation of a cluster of young stars at the northern tip of the ULX bubble may have been triggered by the shock wave itself (perhaps even by a jet), or is just a coincidence.

The ULX bubble is completely dominated by collisional ionization, with no evidence of X-ray photo-ionization; this is inferred in particular from the low [O III] $\lambda 5007$ and He II $\lambda 4686$ emission compared with other bubbles, and relatively high $[\mathrm{Fe} \mathrm{II}] \lambda 1.64 \mu \mathrm{m}$, [S II] $\lambda 6716,6721$, [O I] $\lambda 6300$. The average shock velocity (inferred from the FWHM of the optical emission lines), assumed to be essentially identical to the bulk expansion velocity of the bubble, is $\approx 100-125 \mathrm{~km} \mathrm{~s}^{-1}$ (with likely higher velocities up to $\approx 150 \mathrm{~km}$ $\mathrm{s}^{-1}$ along the north-south direction). This is intermediate between the slower shocks seen in NGC $1313 \mathrm{X}-2\left(\approx 80 \mathrm{~km} \mathrm{~s}^{-1}\right.$ : Pakull \& Mirioni 2002; Weng et al. 2014) and the faster shocks of NGC 7793$\mathrm{S} 26\left(\approx 250 \mathrm{~km} \mathrm{~s}^{-1}\right.$ : Pakull et al. 2010). Consequently, the estimated dynamical age $\left(\approx 6 \times 10^{5} \mathrm{yr}\right)$ is intermediate between the younger NGC 7793-S26 $\left(\approx 2 \times 10^{5} \mathrm{yr}\right)$ and the older NGC $1313 \mathrm{X}-2\left(\approx 8 \times 10^{5}\right.$ yr).

We used three alternative methods (standard bubble theory, $\mathrm{H} \beta$ luminosity and Fe II luminosity) to estimate the long-term-average mechanical power that is inflating the bubble. We found a consistent result of $\sim 2 \times 10^{40} \mathrm{erg} \mathrm{s}^{-1}$. This is higher than the current X-ray luminosity of the central source NGC $5585 \mathrm{X}-1$, as was already noted in other ULX bubbles such as NGC 7793-S26.

The X-1 source itself is by no means an extreme or peculiar example of ULXs. From two XMM-Newton and two Chandra observations, we determined a mildly variable luminosity of $\approx 2-4 \times 10^{39}$ $\mathrm{erg} \mathrm{s}^{-1}$ (assuming isotropic emission), and a spectrum reasonably well fitted with a disk model with peak temperature $k T_{\text {in }} \sim 1.3-1.5$ $\mathrm{keV}$, inner radius $\sim 100 \mathrm{~km}$, and low intrinsic absorption $\left(\lesssim 10^{21}\right.$ $\mathrm{cm}^{-2}$ ). A standard disk-blackbody fit is slightly improved (just at the $90 \%$ confidence level) when we add a (weak) Comptonized powerlaw tail and/or when we allow the radial temperature index $p$ to be $<0.75$ (slim disk scenario). Both corrections have the same effect of making the X-ray spectrum slightly less curved than a standard disk. This type of broadened disk spectrum is often seen in ULXs that are within a factor of 3 above their Eddington luminosity.

We showed that there is a blue, point-like optical counterpart at the X-ray position of X-1, standing out from the other stellar objects in the surrounding few arcsec. The absolute magnitude of this source $\left(M_{V} \approx-4.3 \mathrm{mag}\right)$ is also very typical of ULXs: it is consistent either with a young, massive donor $\operatorname{star}\left(M_{*} / M_{\odot} \approx 20 \pm 5\right.$ if it is a main sequence or subgiant star) or with optical re-emission from the outer rings of an X-ray irradiated disk.

In conclusion, with this study, we have added another interest- ing specimen to the class of shock-ionized nebulae around supercritical accreting X-ray binaries. It is now almost two decades since their nature and importance as a diagnostic of mechanical output power were first recognized (Pakull \& Mirioni 2002). Over this time, we have discovered several new cases, and we have made quantitative progress in their interpretation, refining the derivation of their age and kinetic power from their optical/infrared images and spectra. In forthcoming studies, we will focus on the search for shock-ionized bubbles without an associated central X-ray source: this will constrain the beaming factor and the duty cycle of supercritical accreting sources.

\section{ACKNOWLEDGEMENTS}

RS acknowledges support and hospitality from the Curtin Institute of Radio Astronomy (Perth, Australia) and from the Observatoire de Strasbourg during part of this work. We thank the anonymous referee for their careful reading of the first version of this paper, and their insightful comments and suggestions. We also thank William Blair, Jifeng Liu, Knox Long, Thomas Russell, Paul Sell, Richard White, Frank Winkler for discussions about SNRs and ionized bubbles, and Robert Fesen for the spectral data files from the 1994 Hiltner telescope observations. This paper uses data taken with the MODS spectrographs built with funding from NSF grant AST-9987045 and the NSF Telescope System Instrumentation Program (TSIP), with additional funds from the Ohio Board of Regents and the Ohio State University Office of Research. We used IRAF software for optical analysis: IRAF is distributed by the National Optical Astronomy Observatory, which is operated by the Association of Universities for Research in Astronomy (AURA) under a cooperative agreement with the National Science Foundation. The National Radio Astronomy Observatory is a facility of the National Science Foundation operated under cooperative agreement by Associated Universities, Inc. This research has made use of data obtained from the 3XMM $X M M-N e w t o n$ serendipitous source catalogue compiled by the 10 institutes of the XMM-Newton Survey Science Centre selected by ESA.

\section{DATA AVAILABILITY}

The Chandra, XMM-Newton, HST and VLA datasets used for this work are all available for download from their respective public archives. The $L B T$ data can be provided upon request.

\section{REFERENCES}

Abe Y., Fukazawa Y., Kubota A., Kasama D., Makishima K., 2005, PASJ, 57,629

Abramowicz M.A., Czerny B., Lasota J.P., Szuszkiewicz E., 1988, ApJ, 332, 646

Allen M.G., Groves B.A., Dopita M.A., Sutherland R.S., Kewley L.J., 2008, ApJS, 178, 20

Arnaud K.A., 1996, ASPC, 101, 17

Baldwin A., Phillips M.M., Terlevich R., 1981, PASP, 93, 5

Berghea C.T., Johnson M.C., Secrest N.J., Dudik R.P., Hennessy G.S., Elkhatib A., 2020, ApJ, 896, 117

Blackburn J.K., 1995, in ASP Conf. Ser., Vol. 77, Astronomical Data Analysis Software and Systems IV, ed. R.A. Shaw, H.E. Payne, and J.J.E. Hayes (San Francisco: ASP), 367.

Blair W.P., Long K.S., 1997, ApJS, 108, 261

Caplan J., Deharveng L., 1986, A\&A, 155, 297 
Cappelluti N., et al., 2009, A\&A, 497, 635

Cash W., 1979, ApJ, 228, 939

Cavagnolo K.W., McNamara B.R., Nulsen P.E.J., Carilli C.L., Jones C., Bîrzan L., 2010, ApJ, 720, 1066

Côté S., Carignan C., Sancisi R., 1991, AJ, 102, 904

Cox D.P., Raymond J.C., 1985, ApJ, 298, 651

Cseh D., et al., 2012, ApJ, 749, 17

Dewey D., 2010, SSRv, 157, 229

Dopita M.A., Sutherland R.S., 1995, ApJ 455, 468

Dopita M.A., Sutherland R.S., 1996, ApJS, 102, 161

Dotan C., Shaviv N.J., 2011, MNRAS, 413, 1623

Douna V.M., Pellizza L.J., Laurent P., Mirabel I.F., 2018, MNRAS, 474, 3488

Ebisawa K., Życki P., Kubota A., Mizuno T., Watarai K.-Y., 2003, ApJ, 597, 780

Elvis M., Maccacaro T., Wilson A.S., Ward M.J., Penston M.V., Fosbury R.A.E., Perola G.C., 1978, MNRAS, 183, 129

Epinat B., et al., 2008, MNRAS, 388, 500

Esteban C., García-Rojas J., Carigi L., Peimbert M., Bresolin F., LópezSánchez A.R., Mesa-Delgado A., 2014, MNRAS, 443, 624

Evans I.N., et al., 2010, ApJS, 189, 37

Evans I.N., et al., 2019, HEAD, 114.01

Federrath C., Roman-Duval J., Klessen R.S., Schmidt W., Mac Low M.-M., 2010, A\&A, 512, A81

Feigelson E.D., Berg C.J., 1983, ApJ, 269, 400

Fender R.P., Gallo E., Jonker P.G., 2003, MNRAS, 343, L99

Fender R.P., Belloni T.M., Gallo E., 2004, MNRAS, 355, 1105

Fender R.P., Homan J., Belloni T.M., 2009, MNRAS, 396, 1370

Feng H., Soria R., 2011, NewAR, 55, 166

Fragos T., Lehmer B.D., Naoz S., Zezas A., Basu-Zych A., 2013, ApJ, 776, L31

Fruscione A., et al., 2006, SPIE, 6270, 1

Ganda K., et al., 2007, MNRAS, 380, 506

Garnett D.R., 1992, AJ, 103, 1330

Gierliński M., Done C., Page K., 2008, MNRAS, 388, 753

Gierliński M., Done C., Page K., 2009, MNRAS, 392, 1106

Gladstone J.C., Copperwheat C., Heinke C.O., Roberts T.P., Cartwright T.F., Levan A.J., Goad M.R., 2013, ApJS, 206, 14

Gladstone J.C., Roberts T.P., Done C., 2009, MNRAS, 397, 1836

Godfrey L.E.H., Shabala S.S., 2013, ApJ, 767, 12

Godfrey L.E.H., Shabala S.S., 2016, MNRAS, 456, 1172

Guo F., Mathews W.G., 2012, ApJ, 756, 181

Hartigan P., Morse J.A., Raymond J., 1994, ApJ, 436, 125

Hartigan P., Raymond J., Hartmann L., 1987, ApJ, 316, 323

Heng K., 2010, PASA, 27, 23

Isobe N., Kubota A., Sato H., Mizuno T., 2012, PASJ, 64, 119

Izotov Y.I., Stasińska G., Meynet G., Guseva N.G., Thuan T.X., 2006, A\&A, 448,955

James P.A., et al., 2004, A\&A, 414, 23

Jiang Y.-F., Stone J.M., Davis S.W., 2014, ApJ, 796, 106

Kaaret P., Feng H., Roberts T.P., 2017, ARA\&A, 55, 303

Karachentsev I.D., Makarova L.N., 2019, Astrophysics, 62, 293 (eprint arXiv: 1907.12417)

Kato S., Fukue J., Mineshige S., 1988, in Black-hole accretion disks, S. Kato, J. Fukue, and S. Mineshige Eds., Kyoto University Press (Kyoto, Japan)

Kawaguchi T., 2003, ApJ, 593, 69

Kawashima T., Ohsuga K., Mineshige S., Yoshida T., Heinzeller D., Matsumoto R., 2012, ApJ, 752, 18

King A.R., Pounds K.A., 2003, MNRAS, 345, 657

King A.R., Pounds K.A., 2015, ARA\&A, 53, 115

Kosec P., Pinto C., Fabian A.C., Walton D.J., 2018, MNRAS, 473, 5680

Kubota A., Ebisawa K., Makishima K., Nakazawa K., 2005, ApJ, 631, 1062

Kubota A., Makishima K., 2004, ApJ, 601, 428

Kubota A., Tanaka Y., Makishima K., Ueda Y., Dotani T., Inoue H., Yamaoka K., 1998, PASJ, 50, 667

Leitherer C., et al., 1999, ApJS, 123, 3

Leitherer C., Ekström S., Meynet G., Schaerer D., Agienko K.B., Levesque E.M., 2014, ApJS, 212, 14
Luo B., et al., 2017, ApJSS, 228, 2

Maccacaro T., et al., 1982, ApJ, 253, 504

Madau P., Fragos T., 2017, ApJ, 840, 39

Madau P., Rees M.J., Volonteri M., Haardt F., Oh S.P., 2004, ApJ, 604, 484

Makishima K., Maejima Y., Mitsuda K., Bradt H.V., Remillard R.A., Tuohy I.R., Hoshi R., Nakagawa M., 1986, ApJ, 308, 635

McMullin J.P., Waters B., Schiebel D., Young W., Golap K., 2007, ASPC, 376,127

Marigo P., et al., 2017, ApJ, 835, 77

Mathewson D.S., Clarke J.N., 1973, ApJ, 180, 725

Matonick D.M., Fesen R.A., 1997, ApJS, 112, 49

Mavromatakis F., Boumis P., Goudis C.D., 2003, A\&A, 405, 591

Meier D.L., 2001, ApJ, 548, L9

Middleton M.J., Walton D.J., Roberts T.P., Heil L., 2014, MNRAS, 438, L51

Middleton M.J., et al., 2015, MNRAS, 454, 3134

Mineshige S., Hirano A., Kitamoto S., Yamada T.T., Fukue J., 1994, ApJ, 426, 308

Mirabel I.F., Dijkstra M., Laurent P., Loeb A., Pritchard J.R., 2011, A\&A, 528,149

Mitsuda K., et al., 1984, PASJ, 96, 741

Molina F.Z., Glover S.C.O., Federrath C., Klessen R.S., 2012, MNRAS, 423, 2680

Moon D.-S., Harrison F.A., Cenko S.B., Shariff J.A., 2011, ApJ, 731, L32

Moy E., Rocca-Volmerange B., 2002, A\&A, 383, 46

Narayan R., Sadowski A., Soria R., 2017, MNRAS, 469, 2997

Ogawa T., Mineshige S., Kawashima T., Ohsuga K., Hashizume K., 2017, PASJ, 69, 33

Ohsuga K., Mineshige S., 2011, ApJ, 736, 2

Osterbrock D.E., Ferland G.J., Astrophysics of gaseous nebulae and active galactic nuclei, Sausalito, CA: University Science Books, 2006

Pagel B.E.J., Simonson E.A., Terlevich R.J., Edmunds M.G., 1992, MNRAS, 255,325

Pakull M.W., Grisé F., 2008, AIPC, 1010, 303

Pakull M.W., Mirioni L., 2002, proc. of the symposium 'New Visions of the $\mathrm{X}$-ray Universe in the XMM-Newton and Chandra Era', 26-30 November 2001, ESTEC, The Netherlands (arXiv:astro-ph/0202488)

Pakull M.W., Grisé F., Motch C., 2006, IAUS, 230, 293

Pakull M.W., Soria R., Motch C., 2010, Natur, 466, 209

Parker M.L., et al., 2017, Natur, 543, 83P

Pérez-Montero E., Contini T., 2009, MNRAS, 398, 949

Pilyugin L.S., Thuan T.X., 2005, ApJ, 631, 231

Pinto C., Middleton M.J., Fabian A.C., 2016, Natur, 533, 64

Pinto C., et al., 2017, MNRAS, 468, 2865

Pogge R. W., et al., 2010, SPIE, 7735, 77350A, SPIE.7735

Points S.D., Long K.S., Winkler P.F., Blair W.P., 2019, ApJ, 887, 66

Poutanen J., Lipunova G., Fabrika S., Butkevich A.G., Abolmasov P., 2007, MNRAS, 377, 1187

Rampadarath H., et al., 2018, MNRAS, 476, 2876

Ramsey C.J., Williams R.M., Gruendl R.A., Chen C.-H.R., Chu Y.-H., Wang Q.D., 2006, ApJ, 641, 241

Remillard R.A., McClintock J.E., 2006, ARA\&, 44, 49

Riess A.G., Casertano S., Yuan W., Macri L.M., Scolnic D., 2019, ApJ, 876, 85

Rosen S.R., et al., 2016, A\&A, 590, 1

Russell D.M., Miller-Jones J.C.A., Maccarone T.J., Yang Y.J., Fender R.P., Lewis F., 2011, ApJ, 739, L19

Russell S.C., Dopita M.A., 1992, ApJ, 384, 508

Sarbadhicary S.K., Badenes C., Chomiuk L., Caprioli D., Huizenga D., 2017, MNRAS, 464, 2326

Shakura N.I., Sunyaev R.A., 1973, A\&A, 24, 337

Shimura T., Takahara F., 1995, ApJ, 445, 780

Shrader C.R., Titarchuk L., 2003, ApJ, 598, 168

Soria R., Pakull M.W., Broderick J.W., Corbel S., Motch C., 2010, MNRAS, 409, 541

Steiner J.F., Narayan R., McClintock J.E., Ebisawa K., 2009, PASP, 121, 1279

Sutton A.D., Roberts T.P., Middleton M.J., 2013, MNRAS, 435, 1758 
Sutton A.D., Done C., Roberts T.P., 2014, MNRAS, 444, 2415

Tao L., Feng H., Grisé F., Kaaret P., 2011, ApJ, 737, 81

Tikhonov N.A., Lebedev V.S., Galazutdinova O.A., 2015, AstL, 49, 231

Tombesi F., Meléndez M., Veilleux S., Reeves J.N., González-Alfonso E., Reynolds C.S., 2015, Natur, 519, 436

Traulsen I., et al., 2019, A\&A, 624, 77

Traulsen I., et al., 2020, A\&A, 641, 137

Vierdayanti K., Watarai K.-Y., Mineshige S., 2008, PASJ, 60, 653

Urquhart R.T., Soria R., 2016, ApJ, 831, 56

Walton D.J., et al., 2016, ApJ, 826, L26

Wang Q.D., 2002, MNRAS, 332, 764

Wang S., Liu J., Qiu Y., Bai Y., Yang H., Guo J., Zhang P., 2016, ApJS, 224, 40

Warmels R.H., 1992, ASPC, 25, 115

Watarai K.-Y., Mineshige S., 2003, PASJ, 55, 959

Watarai K.-Y., Fukue J., Takeuchi M., Mineshige S., 2000, PASJ, 52, 133

Watarai K.-Y., Mizuno T., Mineshige S., 2001, ApJ, 549, L77

Weaver R., McCray R., Castor J., Shapiro P., Moore R., 1977, ApJ, 218, 377

Webb N.A., et al., 2020, A\&A, 641, 136

Weng S.-S., Zhang S.-N., Zhao H.-H., 2014, ApJ, 780, 147

Weng S.-S., Feng H., 2018, ApJ, 853, 115

White R.L., Long K.S., Becker R.H., Blair W.P., Helfand D.J., Winkler P.F., 2019, ApJS, 241, 37

Willott C.J., Rawlings S., Blundell K.M., Lacy M., 1999, MNRAS, 309, 1017

Wilms J., Allen A., McCray R., 2000, ApJ, 542, 914

\section{APPENDIX A: LINE WIDTH AND SHOCK VELOCITY}

Inferring a shock velocity from the width of the collisionally ionized optical emission lines is not straightforward, but we can make the following quantitative argument (based on Dewey 2010) with some simplifying assumptions. First, we must distinguish between adiabatic shocks and fully radiative shocks. In the former case, the gas behind the shock picks up a bulk velocity $v_{\text {bulk }}=(3 / 4) v_{\mathrm{s}}$ (i.e., it lags the shock); in the latter case, the shocked gas moves with the shock, $v_{\text {bulk }}=v_{\mathrm{s}}$, and gets swept up in a thin shell. The analysis of Dewey (2010) is mostly focused on adiabatic shocks, in which the post-shock equilibrium temperature is $T \approx(3 / 16)(1 / k) \mu m_{\mathrm{p}} v_{\mathrm{s}}^{2} \approx 3 \times 10^{5} v_{2}^{2} \mathrm{~K}$. Instead, for ULX bubbles, we assume here that it is more appropriate to use the fully radiative shock approximation. Our assumption is justified by the fact that the shocks are not very young, and most of the shocked gas has already recombined and cooled to a range of temperatures $(T \sim 10,000-50,000 \mathrm{~K})$ where it emits low ionisation forbidden lines, such as those we observe from [O I], [S II], [N II] and [Fe II]. In practice, though, we do expect some radiating material also with velocities $(3 / 4) v_{\mathrm{S}} \lesssim v_{\text {bulk }} \lesssim v_{\mathrm{S}}$.

Next, we need to estimate the expansion velocity of the bubble along our line of sight. If we observe the projected central region of a spherical bubble, we see only shocked material coming towards us at $v \approx v_{\text {bulk }}$ (front side of the thin bubble) and material receding at $v \approx-v_{\text {bulk }}$ (back side of the bubble). This corresponds to a velocity distribution function in the central region $f_{\mathrm{c}}(v)=(1 / 2)[\delta(v-$ $\left.\left.v_{\text {bulk }}\right)+\delta\left(v+v_{\text {bulk }}\right)\right]$, where we have used two delta functions for the two projected velocity components, and a mean velocity $\bar{v}=0$. From the velocity distribution, we can then calculate the variance $\sigma_{\mathrm{c}}^{2} \equiv \int v^{2} f_{\mathrm{c}}(v) d v-(\bar{v})^{2}=v_{\text {bulk }}^{2}$. Hence, the root-mean-square $\sigma_{\mathrm{c}}=v_{\text {bulk }}=v_{\mathrm{s}}$, and the Gaussian FWHM observed from the central region is $\mathrm{FWHM}_{\mathrm{c}} \equiv 2 \sqrt{\ln 4} \sigma_{\mathrm{c}} \approx 2.355 v_{\mathrm{s}}$. Finally, we obtain

$v_{\mathrm{S}} \approx 0.425 \mathrm{FWHM}_{\mathrm{c}}$.

In practice, the measured value of $\mathrm{FWHM}_{\mathrm{c}}$ will be a little higher than predicted by the $\delta$-function approximation, both because we are observing a finite (not point-like) region across the centre of the bubble, and because of the likely presence of some cooling gas with velocities $(3 / 4) v_{\mathrm{s}} \lesssim v_{\text {bulk }} \lesssim v_{\mathrm{s}}$. From our analysis of long slit spectra for another shock-ionized bubble, S26 in NGC 7793 (M.W. Pakull et al., in prep.; see also Pakull et al. 2010), we estimate $v_{\mathrm{s}} \approx 0.47 \mathrm{FWHM}_{\mathrm{c}}$. In the adiabatic shock approximation (all the gas at $\left.v_{\text {bulk }}=(3 / 4) v_{\mathrm{s}}\right)$, the relation between the fitted $\mathrm{FWHM}_{\mathrm{c}}$ and the shock velocity is $v_{\mathrm{s}} \approx 0.57 \mathrm{FWHM}_{\mathrm{c}}$ (Dewey 2010), which we can take as an upper limit for our estimate of $v_{\mathrm{s}}$.

Instead of measuring only the lines from central region, we can take spectra for the total emission of the bubble, approximated again as a thin spherical shell with uniform expansion speed of $v_{\text {bulk }}$. In this case, as argued by Dewey (2010), we would observe a (total) uniform distribution function $f_{\mathrm{T}}(v)=1 /\left(2 v_{\text {bulk }}\right)=$ const in radial velocity space, i.e., constant from $v=-v_{\text {bulk }}$ to $v=$ $+v_{\text {bulk }}$. Such a distribution has a mean velocity $\bar{v}=0$. The variance is $\sigma_{\mathrm{T}}^{2} \equiv \int v^{2} f_{\mathrm{T}}(v) d v-(\bar{v})^{2}=(1 / 3) v_{\text {bulk }}^{2}=(1 / 3) v_{\mathrm{s}}^{2}$. Hence, the root-mean-square $\sigma_{\mathrm{T}}=(1 / \sqrt{3}) v_{\mathrm{s}}$, and the Gaussian width is $\mathrm{FWHM}_{\mathrm{T}} \equiv 2 \sqrt{\ln 4} \sigma_{\mathrm{T}} \approx 1.36 v_{\mathrm{s}}$. Finally, we obtain for the full bubble observation

$v_{\mathrm{S}} \approx 0.735 \mathrm{FWHM}_{\mathrm{T}}$.

The equivalent expression for an adiabatic shock is $v_{\mathrm{S}} \approx$ 0.98 $\mathrm{FWHM}_{\mathrm{T}}$ (Dewey 2010; Heng 2010).

In our specific case, we do not have an integral field spectroscopic measurement of the emission from the whole bubble. However, we do have long-slit spectra approximately oriented along the major and minor axes. We can compute an average of the observed Gaussian widths (corrected for instrumental broadening) for a sample of strong lines extracted from the central section of the two slits, as a proxy for $\mathrm{FWHM}_{\mathrm{c}}$. Moreover, we can compute an average intrinsic width of the strongest lines over the whole length of the two slits (obviously excluding the $\mathrm{H}$ II region in the Obs1 slit), as a proxy for $\mathrm{FWHM}_{\mathrm{T}}$.

This paper has been typeset from a $\mathrm{TE}_{\mathrm{E}} \mathrm{X} / \mathrm{L} \mathrm{T} \mathrm{X}$ file prepared by the author. 